\title{
Pacific
}

Journal of

Mathematics

\section{MODULATION AND NATURAL VALUED QUIVER OF AN ALGEBRA}

\author{
FANG LI
}




\title{
MODULATION AND NATURAL VALUED QUIVER OF AN ALGEBRA
}

\author{
FANG LI
}

We generalize the concept of modulation to pseudomodulation and its subclasses including premodulation, generalized modulation and regular modulation. The motivation is to define the valued analogue of natural quiver, called natural valued quiver, of an artinian algebra so as to correspond to its valued Ext-quiver when this algebra is not $k$-splitting over the field $k$. Moreover, we illustrate the relation between the valued Ext-quiver and the natural valued quiver.

The interesting fact we find is that the representation categories of a pseudomodulation and of a premodulation are equivalent respectively to that of a tensor algebra of $\mathscr{A}$-path type and of a generalized path algebra. Their examples are given from two kinds of artinian hereditary algebras. Furthermore, the isomorphism theorem is given for normal generalized path algebras with finite (acyclic) quivers and normal premodulations.

We give four examples of pseudomodulations: first, group species in mutation theory as a seminormal generalized modulation; second, viewing a path algebra with loops as a premodulation with valued quiver that has no loops; third, differential pseudomodulation and its relation with differential tensor algebras; fourth, a pseudomodulation considered as a free graded category.

\section{Introduction}

Throughout this paper, $k$ denotes the ground field.

It is well known that for an artinian $k$-algebra $A$, one has either the Ext-quiver in the case $A$ is $k$-splitting or the valued Ext-quiver otherwise. This quiver $\Gamma$ is used to characterize the structure of $A$ by Gabriel's theorem when $A$ is basic, that is, $A \cong$ $k \Gamma / I$ with admissible ideal $I$ if $A$ is $k$-splitting (e.g., if $k$ is algebraically closed).

Project supported by the National Natural Science Foundation of China (grant numbers 10871170 and 11171296) and the Zhejiang Provincial Natural Science Foundation of China (grant number D7080064) and the Specialized Research Fund for the Doctoral Program of Higher Education of China (grant number 20110101110010).

MSC2010: 16G10, 16G20.

Keywords: pseudomodulation, tensor algebra, natural-valued quiver, valued Ext-quiver, artinian algebra, generalized path algebra. 
Motivated by it, in [Li and Lin 2012], we defined the notion of natural quiver for any artinian algebra in order to construct the analogue of Gabriel's theorem in the case that $A$ is not $k$-splitting and even not basic. This aim has been achieved in the case when $A$ is splitting over radical in [Li and Lin 2012]. The other important piece is the relation between natural quiver and Ext-quiver of a $k$-splitting artinian algebra, which is also given in [ $\mathrm{Li}$ and $\mathrm{Lin} 2012]$.

However, when $A$ is not $k$-splitting, the valued Ext-quiver of $A$ cannot be compared with the natural quiver of $A$. Hence, in the general case, we have to consider these questions:

(i) How should the valued analogue of natural quiver of $A$ be defined so as to correspond to the valued Ext-quiver of $A$ ?

(ii) Following (i), what is the relationship between the valued Ext-quiver and the valued analogue of natural quiver of $A$ ?

The first aim of this paper is to answer these questions. For this, we generalize the concept of modulation to pseudomodulation and its subclasses including premodulation, generalized modulation and regular modulation, in Section 3.

For an artinian algebra $A$, the alteration of natural quiver corresponding to valued Ext-quiver, called natural valued quiver, is introduced via the valued quiver of the corresponding premodulation of $A$. In the case $A$ is basic, we show in Theorem 7.4 that the natural valued quiver is pair-opposite equal to the valued Ext-quiver. Moreover, in Theorem 7.5 we obtain for any artinian algebra $A$ the relation between its natural valued quiver and valued Ext-quiver; this is an improvement of the relation in [ $\mathrm{Li}$ and Lin 2012] between the natural quiver and Ext-quiver in the case $A$ is over an algebraically closed field.

The representation categories of a pseudomodulation and of a premodulation are equivalent respectively to that of a tensor algebra of $\mathscr{A}$-path type and that of a generalized path algebra (Theorem 3.2 and Corollary 5.4). We give their examples from two kinds of artinian hereditary algebras (Corollary 3.3 and Proposition 7.1). In Theorem 5.5, we give the isomorphism theorem for normal generalized path algebras with finite (acyclic) quivers and normal premodulations.

The notion of modulation was introduced in [Dlab 1980; Dlab and Ringel 1976] to characterize representations of a valued quiver over a field $k$, which is not necessarily algebraically closed, using the method of Coxter functors in BernsteinGelfand-Ponomarev theory. This aspect will be discussed for pseudomodulations in follow up work.

Pseudomodulations, as well as generalized path algebras in [Li and Chen 2010; $\mathrm{Li}$ and Lin 2012], can be used as a tool to investigate some properties of structures and representations of an algebra when these properties are not Morita invariants, because the (valued) natural quiver is not Morita invariant. 
Theorem 4.4 characterizes a kind of (semi)normal generalized modulation. In Section 6, we present an interesting example of it from group species in mutation theory [Demonet 2010].

In the theory of mutations [Ladkani 2010; Demonet 2010], it is known that for a finite-dimensional basic hereditary algebra $A \cong k \Gamma$ for a quiver $\Gamma$, under the condition the mutation can be defined, the mutation of $A$ is isomorphic to the path algebra of the quiver that is the mutation of $\Gamma$. Since mutations are perverse equivalent but not Morita equivalent [Ladkani 2010], it is interesting to construct the mutation theory of finite-dimensional (nonbasic in general) algebras via seminormal generalized modulations, using Proposition 6.1.

In Section 6, we first suggest a method for transferring the study on path algebras whose quiver has loops into that on generalized path algebras and premodulations with valued quiver without loops. Second, we still have a notion of differential pseudomodulation and give its relation with differential tensor algebras. Finally, a $k$-pseudomodulation $\mathcal{M}$ and also the related tensor algebra of $\mathscr{A}$-path-type $T(\mathcal{M})$ are equivalently considered as a free graded category $\mathscr{T}$.

\section{Some preliminaries}

2.1. A quiver $Q$ can be understood as two sets $Q_{0}$ and $Q_{1}$ together with a map $Q_{1} \rightarrow Q_{0} \times Q_{0}, a \mapsto(t(a), h(a))$, where $h(a)$ is head of the arrow $a$ and $t(a)$ is the tail of $a$. For each pair $(i, j) \in Q_{0} \times Q_{0}$, we define

$$
\Omega(i, j)=\left\{a \in Q_{1} \mid t(a)=j, h(a)=i\right\} .
$$

Note that $Q_{1}$ is the disjoint union of all $\Omega(i, j)$ for $i, j \in Q_{0}$.

Forgetting the orientation of all arrows in the quiver $Q$, we get the underlying graph of $Q$, which is denoted by $\bar{Q}$.

\subsection{A pseudovalued graph $(\mathscr{G}, \mathscr{D})$ consists of}

(i) a finite set $\mathscr{G}=\{i, j, \ldots\}$ whose elements are called vertices; and

(ii) a correspondence taking any ordered pair $(i, j) \in \mathscr{G} \times \mathscr{G}$ to a nonnegative integer $d_{i j}$ such that if $d_{i j} \neq 0$ then $d_{j i} \neq 0$ for any $(i, j) \in \mathscr{G} \times \mathscr{G}$. If $d_{i j} \neq 0$, such a pair $(i, j)$ is called an edge between the vertices $i$ and $j$, which is written as

$$
\stackrel{\left(d_{i j}, d_{j i}\right)}{i \bullet \bullet j}
$$

If $d_{i j}=d_{j i}=1$, write simply $i \bullet \longrightarrow j$

Of course, $d_{i j}=d_{j i}$ when $i=j$.

The family $\mathscr{D}=\left\{\left(d_{i j}, d_{j i}\right):(i, j) \in \mathscr{G} \times \mathscr{G}\right\}$ is called a valuation of the graph $\mathscr{G}$. 
Due to [Dlab and Ringel 1976; Dlab 1980], if a pseudovalued graph $(\mathscr{G}, \mathscr{D})$ is such that there exist positive integers $\varepsilon_{i}$ (with $i \in \mathscr{G}$ ) such that $d_{i j} \varepsilon_{j}=d_{j i} \varepsilon_{i}$ for all $i, j \in \mathscr{G}$, then $(\mathscr{G}, \mathscr{D})$ is called a valued graph.

An orientation $\Omega$ of a valued or pseudovalued graph $(\mathscr{G}, \mathscr{D})$ is given by prescribing for each edge an ordering, indicated by an oriented edge, that is,

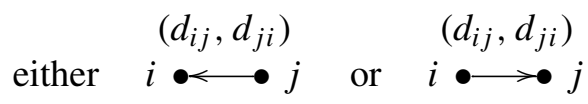

We call a valued or pseudovalued graph with orientation valued or pseudovalued quiver, and denote one by $(\mathscr{G}, \mathscr{D}, \Omega)$.

A vertex $k \in \mathscr{G}$ in the valued quiver $(\mathscr{G}, \mathscr{D}, \Omega)$ is called a sink (or a source) if $i \neq k$ (respectively, $j \neq k$ ) for any oriented edge

$$
\begin{gathered}
\left(d_{i j}, d_{j i}\right) \\
i \bullet{ }^{\longrightarrow} j .
\end{gathered}
$$

A path of the pseudovalued quiver $(\mathscr{G}, \mathscr{D}, \Omega)$ is a sequence $k_{1}, k_{2}, \ldots, k_{t}$ of vertices such that there is a valued oriented edge from $k_{s}$ to $k_{s+1}$ for $s=1,2, \ldots, t-1$. Its length is the number of the valued oriented edges in this path, that is, $t-1$.

2.3. From [Dlab 1980], a $k$-modulation $M=\left(F_{i},{ }_{i} M_{j}\right)$ of a valued graph $(\mathscr{G}, \mathscr{D})$ is a set of division algebras $\left\{F_{i}\right\}_{i \in \mathscr{G}}$ that are finite-dimensional over a common central subfield $k$, together with a set $\left\{_{i} M_{j}\right\}_{i, j \in \varphi}$ of $F_{i}-F_{j}$-bimodules on which $k$ acts centrally, such that $\operatorname{dim}\left({ }_{i} M_{j}\right)_{F_{j}}=d_{i j}, \operatorname{dim}_{F_{i}}\left({ }_{i} M_{j}\right)=d_{j i}$ and ${ }_{j} M_{i}$ is a dual of the bimodule ${ }_{i} M_{j}$ in the sense that we have bimodule isomorphisms

$$
{ }_{j} M_{i} \cong \operatorname{Hom}_{F_{i}}\left({ }_{i} M_{j}, F_{i}\right) \cong \operatorname{Hom}_{F_{j}}\left({ }_{i} M_{j}, F_{j}\right) .
$$

The final isomorphism is from [Dlab 1980, Lemma 0.2]; there is an edge between $i$ and $j$ if and only if ${ }_{i} M_{j}$ and ${ }_{j} M_{i}$ are nonzero.

Now, let $(\mathcal{M}, \Omega)$ be a pair consisting of a $k$-modulation $\mathcal{M}$ of the connected valued graph $(\mathscr{G}, \mathscr{D})$; equivalently say, let $\mathcal{M}=\left(F_{i},{ }_{i} M_{j}\right)$ be a $k$-modulation of a valued quiver $(\mathscr{G}, \mathscr{D}, \Omega)$.

2.4. For the pair $\left(A,{ }_{A} M_{A}\right)$ formed from a $k$-algebra $A$ and an $A$-bimodule $M$, we write the $n$-fold $A$-tensor product $M \otimes_{A} M \otimes \cdots \otimes_{A} M$ as $M^{n}$. Then $T(A, M)=$ $A \oplus M \oplus M^{2} \oplus \cdots \oplus M^{n} \oplus \cdots$ as an abelian group. Let $M^{0}=A$; then $T(A, M)$ becomes a $k$-algebra with multiplication induced by the natural $A$-bilinear maps $M^{i} \times M^{j} \rightarrow M^{i+j}$ for $i \geq 0$ and $j \geq 0$. We call $T(A, M)$ the tensor algebra of $M$ over $A$.

For a $k$-modulation $M=\left(F_{i},{ }_{i} M_{j}\right)$ of a valued quiver $(\mathscr{G}, \mathscr{D}, \Omega)$, we get the tensor

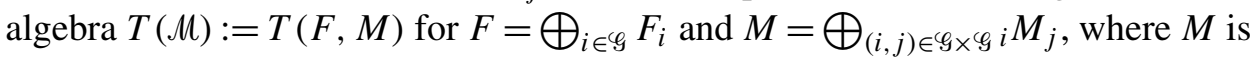
acted on by $F$ as an $F$-F-bimodule through the projection maps $F \rightarrow F_{i}$ for $i \in \mathscr{G}$. 
Representations are interesting because of the following:

Theorem [Dlab 1980]. Let $M=\left(F_{i},{ }_{i} M_{j}\right)$ be a $k$-modulation of a valued quiver $(\mathscr{G}, \mathscr{D}, \Omega)$. Then the category $\operatorname{rep}(\mathcal{M})$ of all finite-dimensional representations is equivalent to the category $\bmod _{T(\mathcal{M})}$ of all finitely generated right $T(\mathcal{M})$-modules.

This result is the generalization of that for the representation category of a finitedimensional path algebra; see [Auslander et al. 1995, Theorem III.1.5].

Modulation and its representations will be generalized in Section 3 such that they are only in the special case with linear spaces over division algebras.

2.5. For two rings $A$ and $B$, the rank of a finitely generated left $A$-module (respectively right $B$-module, $A$ - $B$-bimodule) $M$ is defined as the minimal cardinal number of the sets generators of $M$ as left $A$-module (respectively right $B$-module, $A$ - $B$-bimodule), which is denoted by $\operatorname{rank}_{A} M$ (respectively rank $M_{B}, \operatorname{rank}_{A} M_{B}$ ). Clearly, if $M$ is finitely generated, such rank always exists. As a convention, the rank of the module 0 is said to be 0 .

Let $X=\left\{m_{i}\right\}_{i=1}^{s}$ be the set of generators of a finitely generated $A$ - $B$-bimodule $M$, that is, $M=\sum_{i=1}^{s} A m_{i} B$. If there do not exist $k$-linearly independent sets $\left\{a_{i u} \in A: i=1, \ldots, s ; u=1, \ldots, p\right\} \quad$ and $\quad\left\{b_{i u} \in B: i=1, \ldots, s ; u=1, \ldots, p\right\}$ satisfying $\sum_{i=1, \ldots, s ; u=1, \ldots, p} a_{i u} m_{i} b_{i u}=0$, we say the set $X$ is $A$ - $B$-linearly independent. In this case, we call $M$ a free $A$-B-bimodule with basis $X$.

Clearly, if $M$ is a free $A$ - $B$-bimodule with basis $\left\{m_{i}\right\}_{i=1}^{s}$ and $\left\{b_{j}\right\}_{j=1}^{t}$ is a $k$ basis of $B$ (respectively $\left\{a_{j}\right\}_{j=1}^{t}$ is a $k$-basis of $A$ ), then $M$ is a left free $A$-module with basis $\left\{m_{i} b_{j}\right\}_{i=1, \ldots, s ; j=1, \ldots, t}$ (respectively a right free $B$-module with basis $\left.\left\{a_{j} m_{i}\right\}_{i=1, \ldots, s ; j=1, \ldots, t}\right)$.

Each $A$ - $B$-bimodule $M$ can be realized as a right $B \otimes A^{\text {op }}$-module. So, $M$ is a free $A$-B-bimodule $M$ if and only if $M$ is a free right $B \otimes A^{\mathrm{op}}$-module. In this case, let $M \cong \sum_{i} m_{i}\left(B \otimes A^{\text {op }}\right)$ with basis $\left\{m_{i}\right\}$. Let $\left\{a_{j}\right\}$ be a $k$-basis of $A$. Then $M \cong \sum_{i j} m_{i} a_{j} \otimes B$ as $B$-modules where $m_{i} a_{j}:=a_{j} m_{i}$. It says that $\left\{a_{j} m_{i}\right\}$ is a $B$-basis of $M=\sum_{i} m_{i}\left(B \otimes A^{\mathrm{op}}\right)$.

2.6. Coelho and Liu [2000] introduced the concept of generalized path algebra. Here we review the different but equivalent definition given in [Li and Lin 2012].

Let $Q=\left(Q_{0}, Q_{1}\right)$ be a quiver. Let $\mathscr{A}=\left\{A_{i} \mid i \in Q_{0}\right\}$ be a collection of $k$ algebras with identity $e_{i} \in A_{i}$. Let $A_{0}=\prod_{i \in Q_{0}} A_{i}$ be the direct product $k$-algebra. Clearly, each $e_{i}$ is an orthogonal central idempotent of $A_{0}$. Let

$$
{ }_{i} M_{j}:=A_{i} \Omega(i, j) A_{j}
$$

be the free $A_{i}-A_{j}$-bimodule with basis $\Omega(i, j)$. This is the free $A_{i} \otimes_{k} A_{j}^{\mathrm{op}}$-module over the set $\Omega(i, j)$. Then, the rank of ${ }_{i} M_{j}$ as $A_{i}-A_{j}$-bimodule is just the number 
of arrows from $i$ to $j$ in the quiver $Q$. Thus,

$$
M=\bigoplus_{(i, j) \in Q_{0} \times Q_{0}} A_{i} \Omega(i, j) A_{j}
$$

is an $A_{0}-A_{0}$-bimodule. The generalized path algebra [Coelho and Liu 2000; Li 2007; Li and Lin 2012] is the tensor algebra

$$
T\left(A_{0}, M\right)=\bigoplus_{n=0}^{\infty} M^{\otimes_{A_{0}} n} .
$$

Here $M^{\otimes_{A_{0}} n}=M \otimes_{A_{0}} M \otimes_{A_{0}} \cdots \otimes_{A_{0}} M$ and $M^{\otimes_{A_{0}} 0}=A_{0}$. We denote by $k(Q, \mathscr{A})$ the generalized path algebra. We say $k(Q, \mathscr{A})$ is (semi)normal if all $A_{i}$ are (semi) simple $k$-algebras.

\section{Pseudomodulations and representations of algebras}

As we have seen, modulation essentially is determined by a tensor algebra. From this viewpoint, we will give the notion of pseudomodulation in a more general way. Moreover, we need to restrict the definition of pseudomodulations so as to define some special cases.

Definition. (i) A k-pseudomodulation $\mathcal{M}=\left(A_{i},{ }_{i} M_{j}\right)$ of a pseudovalued graph $(\mathscr{G}, \mathscr{D})$ is defined as a set of artinian $k$-algebras $\left\{A_{i}\right\}_{i \in \mathscr{G}}$, together with a set $\left\{{ }_{i} M_{j}\right\}_{(i, j) \in \mathscr{G}_{\times} \mathscr{G}}$ of finitely generated unital $A_{i}-A_{j}$-bimodules ${ }_{i} M_{j}$ such that $\operatorname{rank}\left({ }_{i} M_{j}\right)_{A_{j}}=d_{i j}$ and $\operatorname{rank}_{A_{i}}\left({ }_{i} M_{j}\right)=d_{j i}$.

(ii) A $k$-pseudomodulation $\mathcal{M}=\left(A_{i},{ }_{i} M_{j}\right)$ of a pseudovalued graph $(\mathscr{G}, \mathscr{D})$ is said to be (semi)normal if all $A_{i}$ for $i \in \mathscr{G}$ are (semi)simple algebras.

(iii) For a $k$-pseudomodulation $\mathcal{M}=\left(A_{i},{ }_{i} M_{j}\right)$ of a valued graph $(\mathscr{G}, \mathscr{D})$, if all ${ }_{i} M_{j}$ are free as $A_{i}-A_{j}$-bimodules, then this pseudomodulation is called a $k$ premodulation.

(iv) If a $k$-pseudomodulation $M=\left(A_{i},{ }_{i} M_{j}\right)$ of a pseudovalued graph $(\mathscr{G}, \mathscr{D})$ is such that

$$
\operatorname{Hom}_{A_{i}}\left({ }_{i} M_{j}, A_{i}\right) \cong \operatorname{Hom}_{A_{j}}\left({ }_{i} M_{j}, A_{j}\right)
$$

as $A_{j}$ - $A_{i}$-bimodules for any $(i, j) \in \mathscr{G} \times \mathscr{G}$, then this pseudomodulation is called a generalized $k$-modulation.

(v) For a generalized modulation $\mathcal{M}=\left(A_{i},{ }_{i} M_{j}\right)$ of a valued graph $(\mathscr{G}, \mathscr{D})$, if all ${ }_{i} M_{j}$ are free as $A_{i}-A_{j}$-bimodules for $i, j \in \mathscr{G}$, then $M=\left(A_{i},{ }_{i} M_{j}\right)$ is called a regular $k$-modulation.

Trivially, a regular $k$-modulation is a generalized modulation and also a premodulation. 
Each ${ }_{i} M_{j}$ is required to be finite generated and ${ }_{i} M_{j} \neq 0$ (meanwhile ${ }_{j} M_{i} \neq 0$ ) if and only if there is an edge between $i$ and $j$ in the (pseudo)valued graph $(\mathscr{G}, \mathscr{D})$.

Example. (i) For a $k$-pseudomodulation $M=\left(F_{i},{ }_{i} M_{j}\right)$ of a pseudovalued graph $(\mathscr{G}, \mathscr{D})$, if each $F_{i}(i \in \mathscr{G})$ is a division $k$-algebra, then $\mathcal{M}=\left(F_{i},{ }_{i} M_{j}\right)$ is just the modulation studied in [Dlab 1980; Dlab and Ringel 1976].

In fact, let $t_{i j}=\operatorname{rank}_{F_{i}} M_{j F_{j}}, \operatorname{dim}_{k} F_{i}=\varepsilon_{i}$, and $\operatorname{dim}_{k} F_{j}=\varepsilon_{j}$. Then $d_{i j}=\operatorname{dim}\left({ }_{i} M_{j}\right)_{F_{j}}=t_{i j} \varepsilon_{i}$ and $d_{j i}=\operatorname{dim}_{F_{i}}\left({ }_{i} M_{j}\right)=t_{i j} \varepsilon_{j}$. Thus $d_{i j} \varepsilon_{j}=d_{j i} \varepsilon_{i}$, which means $(\mathscr{G}, \mathscr{D})$ is a valued graph. The condition $\operatorname{Hom}_{F_{i}}\left({ }_{i} M_{j}, F_{i}\right) \cong$

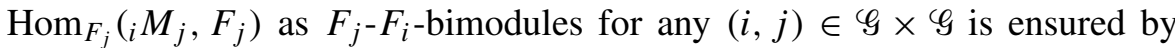
[Dlab 1980, Lemma 0.2].

Hence, the classical modulation in [Dlab 1980; Dlab and Ringel 1976] is a special class of regular modulations.

(ii) In particular, in (i), if $F_{i}$ for $i \in \mathscr{G}$ are finite extension fields of $k$, then $M=$ $\left(F_{i},{ }_{i} M_{j}\right)$ is called a $k$-species of the valued graph $(\mathscr{G}, \mathscr{D})$.

(iii) Moreover, in (i), if the valued graph $(\mathscr{G}, \mathscr{D})$ is given an orientation $\Omega$ and $F_{i}=k$ for $i \in \mathscr{G}$, the bimodule ${ }_{i} M_{j}$ is only a $k$-linear space such that $t_{i j}:=d_{i j}=d_{j i}$ for any pair $(i, j) \in \mathscr{G} \times \mathscr{G}$. Then the valued quiver $(\mathscr{G}, \mathscr{D}, \Omega)$ degenerates to a (nonvalued) quiver $G=\left(G_{0}, G_{1}\right)$ whose arrow number from $i$ to $j$ is just $t_{i j}$ if the pair $(i, j)$ is oriented from $i$ to $j$. Thus, in this case, $T(M)$ is just the path algebra $k G$.

In order to introduce representations of a pseudomodulation, the pseudovalued graph has to be given an orientation as below.

For a $k$-pseudomodulation $M=\left(A_{i},{ }_{i} M_{j}\right)$ over a pseudovalued quiver $(\mathscr{G}, \mathscr{D}, \Omega)$, we define a representation of $\mathcal{M}$ to be an object $\mathscr{V}=\left(V_{i},{ }_{j} \varphi_{i}\right)$, where to each vertex $i \in \mathscr{G}$ corresponds an $A_{i}$-module $V_{i}$ and to each oriented edge $i \rightarrow j$ corresponds an $A_{j}$-homomorphism ${ }_{j} \varphi_{i}: V_{i} \otimes_{A_{i} i} M_{j} \rightarrow V_{j}$. If each $V_{i}$ is finitely generated as $A_{i}$-module, this representation $\mathscr{V}=\left(V_{i},{ }_{j} \varphi_{i}\right)$ is said to be finitely generated.

If in the definition above we take the case that $A_{i}=A_{j}=k$ and let $\operatorname{dim}_{k i} M_{j}=t_{i j}$, then $d_{i j}=d_{j i}=t_{i j}$ and we get a representation $\mathscr{V}=\left(V_{i},{ }_{j} \varphi_{i}\right)$ of the nonvalued quiver $G$ (that is, a representation of $k G$ ) with ${ }_{j} \varphi_{i}: V_{i} \rightarrow V_{j}$.

A morphism $\alpha$ from a representation $\mathscr{V}=\left(V_{i},{ }_{j} \varphi_{i}\right)$ to another representation $\mathcal{U}=\left(U_{i},{ }_{j} \psi_{i}\right)$ consists of $A_{i}$-module homomorphisms $\alpha_{i}: V_{i} \rightarrow U_{i}$ for all $i \in \mathscr{G}$ preserving the structure of the objects, that is, those such that all diagrams

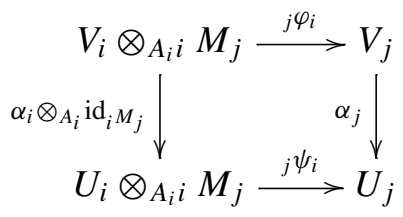

commute for each oriented edge $i \rightarrow j$. 
Let $\operatorname{Rep}(\mathcal{M})$ (respectively $\operatorname{rep}(\mathcal{M})$ ) be the category consisting of all (respectively finitely generated) representations of $\mathcal{M}$.

For a $k$-pseudomodulation $M=\left(A_{i},{ }_{i} M_{j}\right)$ of a pseudovalued quiver $(\mathscr{G}, \mathscr{D}, \Omega)$, we obtain the tensor algebra $T(M):=T(A, M)$ for $A=\bigoplus_{i \in \mathscr{G}} A_{i}$ and $M=$ $\bigoplus_{(i, j) \in \mathscr{G}_{\times} \varphi_{i}} M_{j}$, where $M$ is acted on by $A$ as an $A$-A-bimodule through the projection maps $A \rightarrow A_{i}$ for $i \in \mathscr{G}$.

Conversely, consider a tensor algebra $T(A, M)$ with $A=\bigoplus_{i \in I} A_{i}$ and $M=$ $\bigoplus_{(i, j) \in I \times I}{ }_{i} M_{j}$ and subalgebras $A_{i}$ and $A_{i}-A_{j}$-bimodules ${ }_{i} M_{j}$ for $i, j \in I$. Let $d_{i j}=\operatorname{rank}\left({ }_{i} M_{j}\right)_{A_{j}}$ and $d_{j i}=\operatorname{rank}_{A_{i}}\left({ }_{i} M_{j}\right)$. Let $\mathscr{D}=\left\{d_{i j}, d_{j i}:(i, j) \in I \times I\right\}$ and $\varphi=I$. For any ${ }_{i} M_{j} \neq 0$, give an oriented edge from $i$ to $j$. Then we get a pseudovalued quiver $(\mathscr{G}, \mathscr{D}, \Omega)$ and a $k$-pseudomodulation $\mathcal{M}=\left(A_{i},{ }_{i} M_{j}\right)$.

We call such a tensor algebra as above an A-path-type tensor algebra [Li 2007] on the pseudovalued quiver $(\mathscr{G}, \mathscr{D}, \Omega)$.

Therefore:

Proposition 3.1. In the way described above, pseudomodulations and tensor algebras of A-path type with finitely generated bimodules can be constructed one from another.

Clearly, representations of the classical modulations and their morphisms in [Dlab 1980; Dlab and Ringel 1976] are respectively the special cases of that of pseudomodulations and their morphisms given here.

The next result about $k$-pseudomodulation generalizes Dlab's theorem $(\S 2.4)$ :

Theorem 3.2. Let $\mathcal{M}=\left(A_{i},{ }_{i} M_{j}\right)$ be a $k$-pseudomodulation of a pseudovalued quiver $(\mathscr{G}, \mathscr{D}, \Omega)$. Then the category $\operatorname{Rep}(\mathcal{M})$ of all representations of $\mathcal{M}$ is equivalent to the category $\operatorname{Mod}_{T(M)}$ of right $T(M)$-modules. Similarly the category $\operatorname{rep}(\mathcal{M})$ of finitely generated representations of $\mathcal{M}$ is equivalent to the category $\bmod _{T(\mathcal{M})}$ of finitely generated $T(M)$-modules.

Proof. Let $\mathscr{V}=\left(V_{i},{ }_{j} \varphi_{i}\right)$ be a representation of $\mathcal{M}$. Define the corresponding right $T(M)$-module $V$ as follows.

Let $V=\bigoplus_{i \in \mathscr{G}} V_{i}$. Firstly, the right $A$-action on $V$ is given via the projections $A \rightarrow A_{i}$ for $i \in \mathscr{G}$, and then the right $M$-action on $V$ is defined by the ${ }_{j} \varphi_{i}$, that is, for the oriented edge $i \rightarrow j$, we put $v_{i} m_{i j}={ }_{j} \varphi_{i}\left(v_{i} \otimes m_{i j}\right)$ for $v_{i} \in V_{i}$ and $m_{i j} \in{ }_{i} M_{j}$, and then extend by distributivity; finally, the $T(M)$-action on $V$ is determined inductively in a unique way by the $M$-action, that is,

$$
v_{i}\left(m_{i j} \otimes \cdots \otimes m_{p q} \otimes m_{q s}\right)={ }_{s} \varphi_{q}\left(\left(v_{i}\left(m_{i j} \otimes \cdots \otimes m_{p q}\right)\right) \otimes m_{q s}\right) .
$$

Thus, $V$ becomes a $T(M)$-module.

If $\alpha$ is a morphism of representations from $\mathscr{V}$ to $\mathcal{U}$, then we can define the $T(\mathcal{M})$ module morphism $\bar{\alpha}$ from $V$ to $U$ with $\bar{\alpha}\left(\bigoplus_{i \in \mathscr{G}} v_{i}\right)=\bigoplus_{i \in \mathscr{G}} \alpha_{i}\left(v_{i}\right)$. Thus, we get 
the functor $F: \operatorname{Rep}(\mathcal{M}) \rightarrow \bmod _{T(\mathcal{M})}$ with $F(\mathscr{V})=V$ and $F(\alpha)=\bar{\alpha}$. In fact, for $\alpha: \mathscr{V} \rightarrow \mathcal{U}$ and $\beta: \mathcal{U} \rightarrow \mathcal{W}$, we have $\beta \cdot \alpha=\left\{\beta_{i} \alpha_{i}: i \in \mathscr{G}\right\}$; then $F(\beta \cdot \alpha)=F(\beta) \cdot F(\alpha)$.

Conversely, we can define the inverse functor $G$. For $V \in \operatorname{Mod}_{T(\mathcal{M})}$, let $V_{i}=V A_{i}$. Then $V=V A=\bigoplus_{i \in \mathscr{G}} V A_{i}=\bigoplus_{i \in \mathscr{G}} V_{i}$. When there is an oriented edge $i \rightarrow j$, we have ${ }_{i} M_{j} \neq 0$. In general, $V_{i} \cdot{ }_{i} M_{j}=V A_{i} \cdot{ }_{i} M_{j}=V \cdot{ }_{i} M_{j} A_{j} \subset V A_{j}=V_{j}$. Then, we can induce the $A_{j}$-module morphisms ${ }_{j} \varphi_{i}: V_{i} \otimes_{A_{i} i} M_{j} \rightarrow V_{j}$ under this $M$-action. Thus, by the definition, $\mathscr{V}=\left(V_{i},{ }_{j} \varphi_{i}\right)$ is a representation of $\mathcal{M}$, that is, $\mathscr{V} \in \operatorname{Rep}(M)$.

For $V, U \in \operatorname{Mod}_{T(\mathcal{M})}$ and $\bar{\alpha}: V \rightarrow U$ a $T(\mathcal{M})$-homomorphism, let $\alpha_{i}=\left.\bar{\alpha}\right|_{V_{i}}$. Then $\alpha_{i}\left(V_{i}\right)=\alpha_{i}\left(V A_{i}\right)=\bar{\alpha}(V) A_{i} \subset U A_{i}=U_{i}$. From the $T(M)$-linearity of $\bar{\alpha}$, the commutative diagram

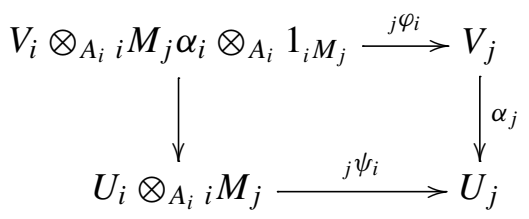

follows for each oriented edge $i \rightarrow j$, where ${ }_{j} \psi_{i}$ is defined as similarly as ${ }_{j} \varphi_{i}$. So, $\alpha=\left\{\alpha_{i}: i \in \mathscr{G}\right\}$ is a morphism from $\mathscr{V}$ to $\mathcal{U}$ in $\operatorname{Rep}(\mathcal{M})$. Define the functor $G$ satisfying $G(V)=\mathscr{V}$ and $G(\bar{\alpha})=\alpha$. For $\bar{\alpha}: V \rightarrow U$ and $\bar{\beta}: U \rightarrow W$, it follows that $\bar{\alpha}=\bigoplus_{i \in \mathscr{G}} \alpha_{i}$ and $\bar{\beta}=\bigoplus_{i \in \mathscr{G}} \beta_{i}$. Then, $\bar{\beta} \cdot \bar{\alpha}=\bigoplus_{i \in \mathscr{G}} \beta_{i} \alpha_{i}$. Hence, $G(\bar{\beta} \cdot \bar{\alpha})=\left\{\beta_{i} \alpha_{i}: i \in \mathscr{G}\right\}=\beta \cdot \alpha=G(\bar{\beta}) \cdot G(\bar{\alpha})$.

Obviously, $F$ and $G$ are mutual-inverse equivalence functors between $\operatorname{Rep}(M)$ and $\operatorname{Mod}_{T(\mu)}$.

Drozd and Kirichenko [1994] proved for a finite-dimensional algebra $A$ with radical $r$ that if the quotient algebra $A / r$ is separable, then $A$ is isomorphic to a quotient algebra of $T\left(A / r, r / r^{2}\right)$ by an admissible ideal $I$, that is, $J^{s} \subset I \subset J^{2}$ for a positive integer $s$.

Moreover, if this algebra $A$ is hereditary, then $I=0$ such that $A \cong T\left(A / r, r / r^{2}\right)$. Let $A / r=\bigoplus_{i=1}^{s} A_{i}$, where $A_{i}$ are simple ideals of $A / r$. Then, $r / r^{2}$ is an $A / r$ $A / r$-bimodule with natural left and right module actions. Let an $A_{i}$ - $A_{j}$-bimodule ${ }_{i} M_{j}$ equal $A_{i} r / r^{2} A_{j}$ for any $i, j=1, \ldots s$. By Proposition 3.1, the corresponding pseudomodulation $M=\left(A_{i},{ }_{i} M_{j}\right)$ of a pseudovalued quiver $(\mathscr{G}, \mathscr{D}, \Omega)$ can be constructed from this tensor algebra $T\left(A / r, r / r^{2}\right)$, which is called the related pseudomodulation of the finite-dimensional hereditary A. Therefore, by Theorem 3.2:

Corollary 3.3. Let $A$ be a finite-dimensional hereditary algebra with radical $r$ and let $M=\left(A_{i},{ }_{i} M_{j}\right)$ be its related pseudomodulation of a pseudovalued quiver $(\mathscr{G}, \mathscr{D}, \Omega)$. If $A / r$ is separable, then the representation category $\operatorname{Rep}(M)$ is equivalent to the module category $\operatorname{Mod}_{A}$, and likewise for the finitely generated $\operatorname{rep}(\mathcal{M})$ and $\bmod _{A}$. 


\section{A kind of generalized $k$-modulations}

By the Wedderburn-Artin theorem, the center of a semisimple algebra $A$ over an algebraically closed field $k$ is just the field $k$. Define $\mu: A \rightarrow \operatorname{End}_{k}(A)$ with $\mu(a)=\rho_{a}$, where $\rho_{a}$ is the right translation on $A$ by the right multiplication of $a$. Obviously, $\rho_{a} \in \operatorname{End}_{k}(A)$. It is easy to check that $\mu$ is a monomorphism of algebras.

Define $t: A \rightarrow k$ with $t(a)=\operatorname{tr}(\mu(a))$. Then, $t$ is the character of the right regular representation of $A$ satisfying that $t(a b)=t(b a)$ for any $a, b \in A$. In fact, trivially, $t$ is $k$-linear and $t(a b)=\operatorname{tr}(\mu(a b))=\operatorname{tr}(\mu(a) \mu(b))=\operatorname{tr}(\mu(b) \mu(a))=t(b a)$.

Lemma 4.1. Let $A$ be a finite-dimensional simple $k$-algebra with $k$ algebraically closed and having characteristic satisfying char $k \nmid \sqrt{\operatorname{dim}_{k} A}$. For any $a \neq 0$ in $A$, we have $t(a A) \neq 0$.

Proof. By the Wedderburn-Artin theorem, $A \cong M_{n}(k)$, the $n \times n$ full matrix algebra over $k$ for $n=\sqrt{\operatorname{dim}_{k} A}$. For simplicity, we think of $a$ as a nonzero $n \times n$ matrix and as right ideal of $A$; then $a A \neq 0$ consists of all $n \times n$ matrices over $k$ whose rows are all 0 except for some $i_{1}, i_{2}, \ldots, i_{s}$-rows. Choose a matrix $X=E_{i_{1} i_{1}}$ in $a A$ with the element 1 in position $\left(i_{1}, i_{1}\right)$ and 0 in all other positions. Then under the $k$-basis $\left\{E_{i j}\right\}_{i, j=1}^{n}$ of $A$, we have $t(X)=\operatorname{tr} \mu(X)=n \cdot 1 \neq 0$ since char $k \nmid n$. Therefore, we have $t(a A) \neq 0$.

Lemma 4.2. Let $A$ and $B$ be finite-dimensional simple $k$-algebras with $k$ algebraically closed such that char $k \nmid \sqrt{\operatorname{dim}_{k} A \operatorname{dim}_{k} B}$. Then, for an $A$-B-bimodule $M$, we have $\operatorname{Hom}_{A}(M, A) \cong \operatorname{Hom}_{B}(M, B)$ as $B$-A-bimodules.

Proof. First, we prove $\operatorname{Hom}_{A}\left(M,{ }_{A} A_{A}\right) \cong \operatorname{Hom}_{k}(M, k)$ as $B$-A-bimodules, where $\operatorname{Hom}_{k}(M, k)$ consists of all $k$-homomorphism with the bimodule structure defined by $(b \psi a)(m)=\psi(a m b)$ for $a \in A, b \in B, m \in M$ and $\psi \in \operatorname{Hom}_{k}(M, k)$.

Indeed, for $b_{1}, b_{2} \in B$ and $m \in M$,

$$
\left(\left(b_{1} b_{2}\right) \psi\right)(m)=\psi\left(m b_{1} b_{2}\right)=\psi\left(\left(m b_{1}\right) b_{2}\right)=\left(b_{2} \psi\right)\left(m b_{1}\right)=\left(b_{1}\left(b_{2} \psi\right)\right)(m) ;
$$

then $\left(b_{1} b_{2}\right) \psi=b_{1}\left(b_{2} \psi\right)$, and similarly $\psi\left(a_{1} a_{2}\right)=\left(\psi a_{1}\right) a_{2}$ for $a_{1}, a_{2} \in A$.

Now, define the map $\tau: A \rightarrow \operatorname{Hom}_{k}(A, k)$ by $\tau(a)=a t$ for $a \in A$, where $a t \in \operatorname{Hom}_{k}(A, k)$ by $(a t)(x)=t(a x)$ for $x \in A$. Obviously, $\tau$ is $k$-linear.

Moreover, $\tau$ is injective. In fact, for $a \in \operatorname{ker} \tau$, it means that $(a t)(x)=0$ for any $x \in A$; then $t(a x)=0$, or say, $a A \subset \operatorname{ker} t$ for the right ideal $a A$ of $A$, which is equivalent to $t(a A)=\operatorname{tr} \mu(a A)=0$. Thus, $a=0$ by Lemma 4.1. Hence, $\operatorname{ker} \tau=0$.

Since $\operatorname{dim}_{k} A=\operatorname{dim}_{k} \operatorname{Hom}_{k}(A, k)$ are finite, $\tau$ is a $k$-linear isomorphism.

Similarly, define $t a \in \operatorname{Hom}_{k}(A, k)$ by $(t a)(x)=t(x a)$ for $x \in A$. Since $t(a x)=$ $t(x a)$ for any $a, x \in A$, we get $\tau(a)=a t=t a$. It follows that ${ }_{A} A_{A} \cong \operatorname{Hom}_{k}(A, k)$ via $\tau$ as $A$-A-bimodules. Thus, $\operatorname{Hom}_{A}\left(M,{ }_{A} A_{A}\right) \cong \operatorname{Hom}_{A}\left(M, \operatorname{Hom}_{k}(A, k)\right) \cong$ $\operatorname{Hom}_{k}\left(A \otimes_{A} M, k\right) \cong \operatorname{Hom}_{k}(M, k)$ as $B$ - $A$-bimodules, as required. 
Similarly, $\operatorname{Hom}_{B}\left(M,{ }_{B} B_{B}\right) \cong \operatorname{Hom}_{k}(M, k)$ holds as $B$ - $A$-bimodules. Therefore, we have $\operatorname{Hom}_{A}\left(M,{ }_{A} A_{A}\right) \cong \operatorname{Hom}_{B}\left(M,{ }_{B} B_{B}\right)$.

This lemma improves [Dlab 1980, Lemma 0.2].

Trivially, the condition in Lemma 4.2 is always satisfied if the field $k$ is algebraically closed of characteristic 0 .

Lemma 4.3. Let $A$ and $B$ be finite-dimensional semisimple algebras over an algebraically closed, characteristic 0 field $k$. Then, for an $A$-B-bimodule $M$, we have $\operatorname{Hom}_{A}(M, A) \cong \operatorname{Hom}_{B}(M, B)$ as B-A-bimodules.

Proof. Let $A=\bigoplus_{i=1}^{s} A_{i}$ and $B=\bigoplus_{j=1}^{t} B_{j}$ with simple ideals $A_{i}$ and $B_{j}$. Then

$$
\begin{aligned}
\operatorname{Hom}_{A}(M, A) & \cong \bigoplus_{i=1}^{s} \operatorname{Hom}_{A}\left(A_{i} M, A_{i}\right) \cong \bigoplus_{i=1}^{s} \operatorname{Hom}_{A_{i}}\left(A_{i} M, A_{i}\right) \\
& \cong \bigoplus_{i=1}^{s} \bigoplus_{j=1}^{t} \operatorname{Hom}_{A_{i}}\left(A_{i} M B_{j}, A_{i}\right) \cong \bigoplus_{i=1}^{s} \bigoplus_{j=1}^{t} \operatorname{Hom}_{B_{j}}\left(A_{i} M B_{j}, B_{j}\right) \\
& \cong \bigoplus_{j=1}^{t} \operatorname{Hom}_{B_{j}}\left(A M B_{j}, B_{j}\right) \cong \operatorname{Hom}_{B}(M, B)
\end{aligned}
$$

Applying Lemma 4.3 to $A_{i}$ and ${ }_{i} M_{j}$ below and taking the definitions on page 110 into account, we obtain:

Theorem 4.4. Let $M=\left(A_{i},{ }_{i} M_{j}\right)$ be a pseudomodulation of a pseudovalued graph $(\mathscr{G}, \mathfrak{D})$ over an algebraically closed field $k$ of characteristic 0 . If all $A_{i}$ for $i \in \mathscr{G}$ are (semi)simple algebras, then $\mathcal{M}$ is a (semi)normal generalized modulation.

From this theorem and its proof, we see that condition (3), which is required by the definition of the classical modulation in Section 2, is not always true for pseudomodulations.

A pseudovalued quiver of a pseudomodulation is an analogue of natural quiver of its corresponding tensor algebra of $\mathscr{A}$-path type, similar to that of a generalized path algebra; see Section 5 and Section 7.

\section{Premodulations and generalized path algebras}

In this section, we give some premodulations and their applications to generalized path algebras and artinian algebras.

As an generalization of path algebras, normal generalized path algebras were used in [ $\mathrm{Li}$ 2007; Li and Chen 2010; Li and Lin 2012; Li and Wen 2008] to characterize the structure and representations of artinian algebras via the method of natural quivers. This, unlike the classical method, does not depend on the corresponding basic algebras. 
In [Dlab 1980; Dlab and Ringel 1976], $k$-representation types of valued quivers were classified through the corresponding relations between valued quivers and $k$ modulations. We will see that the corresponding relationship still holds between (semi)normal generalized path algebras and (semi)normal regular $k$-modulations.

Lemma 5.1. Let $k(Q, A)$ be a generalized path algebra, let $M$ and ${ }_{i} M_{j}$ defined as in (1) and (2), let $\varepsilon_{i}=\operatorname{dim}_{k} A_{i}$, and let $d_{i j}=\operatorname{rank}\left({ }_{i} M_{j}\right)_{A_{j}}$ and $d_{j i}=\operatorname{rank}_{A_{i}}\left({ }_{i} M_{j}\right)$ for all $i, j \in Q_{0}$. Then, $d_{i j} \varepsilon_{j}=d_{j i} \varepsilon_{i}$ for any $i, j \in Q_{0}$.

Proof. Let $\left\{m_{l}\right\}_{l \in \Lambda}$ be an $A_{i}-A_{j}$-basis of ${ }_{i} M_{j}$ as a free $A_{i}-A_{j}$-bimodule. Let $\left\{a_{s}\right\}_{s \in \Phi}$ and $\left\{b_{t}\right\}_{t \in \Psi}$ be respectively $k$-bases of $A_{i}$ and $A_{j}$. Then, ${ }_{i} M_{j}$ is right $A_{j}$-free and left $A_{i}$-free with $A_{j}$-basis $\left\{a_{s} m_{l}\right\}_{s \in \Phi, l \in \Lambda}$ and $A_{i}$-basis $\left\{m_{l} b_{t}\right\}_{l \in \Lambda, t \in \Psi}$, respectively. Thus, $|\Phi|=\varepsilon_{i},|\Psi|=\varepsilon_{j}$, and $|\Phi||\Lambda|=d_{i j}$ and $|\Lambda||\Psi|=d_{j i}$. So, $|\Lambda|=d_{i j} / \varepsilon_{i}=$ $d_{j i} / \varepsilon_{j}$. It follows that $d_{i j} \varepsilon_{j}=d_{j i} \varepsilon_{i}$.

By this lemma, we can get the valued quiver $\left(Q_{0}, \mathscr{D}, \Omega\right)$, the induced valued quiver from $k(Q, \mathscr{A})$; it has valuation $\mathscr{D}=\left\{\left(d_{i j}, d_{j i}\right):(i, j) \in Q_{0} \times Q_{0}\right\}$ and there is just a unique oriented edge from $i$ to $j$ when ${ }_{i} M_{j} \neq 0$.

By the definition on page 110 and Theorem 4.4:

Proposition 5.2. Let $k(Q, A)$ be a generalized path algebra over a field $k$ and let $M$ and ${ }_{i} M_{j}$ be defined as in (1) and (2).

(i) A k-premodulation $M=\left(A_{i},{ }_{i} M_{j}\right)$ is obtained from the induced valued quiver $\left(Q_{0}, \mathscr{D}, \Omega\right)$ with $d_{i j}=\operatorname{rank}\left({ }_{i} M_{j}\right)_{A_{j}}$ and $d_{j i}=\operatorname{rank}_{A_{i}}\left({ }_{i} M_{j}\right)$ for the valuation $\mathscr{D}=\left\{\left(d_{i j}, d_{j i}\right):(i, j) \in Q_{0} \times Q_{0}\right\}$;

(ii) If $k$ is an algebraically closed field of characteristic 0 and $k(Q, A)$ is seminormal, then if ${ }_{i} M_{j} \neq 0$ (that is, if there exists an arrow from $i$ to $j$ ), then $\operatorname{Hom}\left({ }_{i} M_{j}, A_{i}\right)_{A_{i}} \cong \operatorname{Hom}\left({ }_{i} M_{j}, A_{j}\right)_{A_{j}}$ by Theorem 4.4, which means that in this case, $\mathcal{M}=\left(A_{i},{ }_{i} M_{j}\right)$ is a regular modulation.

By definition, such a $k$-premodulation $M=\left(A_{i},{ }_{i} M_{j}\right)$ built from the $\mathscr{A}$-path algebra $k(Q, \mathscr{A})$ is unique. We call it the corresponding $k$-premodulation of $k(Q, \mathscr{A})$, and denote it by $\mathcal{M}_{k(Q, \mathscr{A})}$; its valued quiver is just the induced valued quiver from $k(Q, \mathscr{A})$.

Conversely, given a $k$-premodulation $M=\left(A_{i},{ }_{i} M_{j}\right)$ of a valued quiver $(\mathscr{G}, \mathscr{D}, \Omega)$ with semisimple algebras $A_{i}(i \in \mathscr{G})$, we illustrate how to build its generalized path algebra. In fact, we only need to set up the quiver $Q$ for a generalized path algebra. Let the vertex set $Q_{0}=\mathscr{G}$. For any oriented pair $(i, j) \in \mathscr{G}_{\times} \mathscr{G}$, let $t_{i j}$ be the number of generators in the $A_{i}-A_{j}$-basis of ${ }_{i} M_{j}$ as a free $A_{i}-A_{j}$-bimodule and set $t_{i j}$ arrows from $i$ to $j$. Then the arrow set $Q_{1}$ is given when the oriented pair $(i, j)$ runs over the whole set $\mathscr{G} \times \mathscr{G}$. Thus the quiver $Q$ is constructed and then the normal path algebra $k(Q, \mathscr{A})=T\left(A_{0}, M\right)$ is obtained, where $M=\bigoplus_{i, j} A_{i} \Omega(i, j) A_{j}$ and $A_{0}=\prod_{i \in Q_{0}} A_{i}$. 
Since ${ }_{i} M_{j}$ and $A_{i} \Omega(i, j) A_{j}$ have the same numbers of generators in their bases as free $A_{i}$ - $A_{j}$-bimodules, we get ${ }_{i} M_{j} \cong A_{i} \Omega(i, j) A_{j}$ for any $(i, j) \in \mathscr{G} \times \mathscr{G}$ following the invariant basis property of all $A_{i}$ as semisimple algebras. Hence, the premodulation constructed from $k(Q, \mathscr{A})$ in the way of Proposition 5.2 is just $M=\left(A_{i},{ }_{i} M_{j}\right)$.

Thus, we have the following:

Theorem 5.3. Premodulations and generalized path algebras can be constructed one from another in the way described above. When the field $k$ is algebraically closed of characteristic 0, (semi)normal premodulations are (semi)normal regular modulations.

By this and Theorem 3.2, we have:

Corollary 5.4. For a generalized path algebra $k(Q, A)$ and the corresponding $k$ premodulation $\mathcal{M}=\left(A_{i},{ }_{i} M_{j}\right)$, the categories $\operatorname{Rep}(M)$ and $\operatorname{rep}(M)$ are equivalent to the categories $\operatorname{Mod}_{k(Q, \&)}$ and $\bmod _{k(Q, \&)}$, respectively.

More concretely, using the functors in the proof of Theorem 3.2, we can give the mutual constructions between representations of a generalized path algebra $k(Q, \mathscr{A})$ and that of its corresponding $k$-premodulation $M=\left(A_{i},{ }_{i} M_{j}\right)$.

We say two $k$-pseudomodulations $M=\left(A_{i},{ }_{i} M_{j}\right)$ of the pseudovalued quiver $\left(Q_{0}, \mathscr{D}, \Omega\right)$ and $\mathcal{N}=\left(B_{i},{ }_{i} N_{j}\right)$ of $\left(P_{0}, \mathscr{C}, \Psi\right)$ are isomorphic if there exists a permutation $\theta$ such that $\left(Q_{0}, \mathscr{D}, \Omega\right) \cong\left(P_{0}, \mathscr{C}, \Psi\right)$ via $\theta$ as pseudovalued quivers, $A_{i} \cong B_{\theta(i)}$ as $k$-algebras, and ${ }_{i} M_{j} \cong{ }_{\theta(i)} N_{\theta(j)}$ as bimodules for any $(i, j) \in Q_{0} \times Q_{0}$. Here, " $\left(Q_{0}, \mathscr{D}, \Omega\right) \cong\left(P_{0}, \mathscr{C}, \Psi\right)$ via $\theta$ as pseudovalued quivers" means that they are isomorphic via a permutation $\theta$ as directed graphs and $d_{i j}=c_{\theta(i) \theta(j)}$ and $d_{j i}=c_{\theta(j) \theta(i)}$ for any $(i, j) \in Q_{0} \times Q_{0}$.

Although a pseudomodulation and tensor algebra of $\mathscr{A}$-path type can be constructed one after another as stated in Proposition 3.1, the isomorphism condition cannot be shifted between them. In fact, if two pseudomodulations are isomorphic, then their related tensor algebras are isomorphic too, but the converse is not true.

For example, let $\Delta$ be a quiver consisting of a unique vertex without loops and $\Delta^{\prime}$ be a quiver consisting of two vertices without loops and arrows. Clearly $\Delta ¥ \Delta^{\prime}$. For any two artinian algebras $S_{1}$ and $S_{2}$, we have $k\left(\Delta,\left\{S_{1} \oplus S_{2}\right\}\right) \cong S_{1} \oplus S_{2} \cong$ $k\left(\Delta^{\prime},\left\{S_{1}, S_{2}\right\}\right)$. However, trivially, their related premodulations $\mathcal{M}_{k\left(\Delta,\left\{S_{1} \oplus S_{2}\right\}\right)}$ and $M_{k\left(\Delta^{\prime},\left\{S_{1}, S_{2}\right\}\right)}$ are not isomorphic.

This example means that the isomorphism theorem does not hold for generalized path algebras in general. Now, we give some special cases of generalized path algebras in which it does hold.

(i) The path algebras $k Q$ and $k P$ are isomorphic if and only if $Q \cong P$ as quivers [Liu et al. 1986]. 
(ii) If the finite quivers $\Delta$ and $\Delta^{\prime}$ are acyclic, the normal generalized path algebras $k(\Delta, \mathscr{A})$ and $k\left(\Delta^{\prime}, \mathscr{A}^{\prime}\right)$ are isomorphic if and only if there is an isomorphism $\Delta \cong$ $\Delta^{\prime}$ via $\theta$ as quivers such that $A_{i} \cong A_{\theta(i)}^{\prime}$ as algebras for $i \in Q_{0}$ [Chen 2008].

(iii) When $\Delta$ and $\Delta^{\prime}$ have oriented cycles, the isomorphism theorem for $k(\Delta, \mathscr{A})$ and $k\left(\Delta^{\prime}, \mathscr{A}^{\prime}\right)$ as in (ii) can also be proved using the method proving (i) in [Liu et al. 1986] or the dual method for generalized path coalgebras given in [ $\mathrm{Li}$ and Liu 2008].

In summary:

Theorem 5.5 (isomorphism theorem). Two normal generalized path algebras with finite (acyclic) quivers are isomorphic if and only if their corresponding normal $k$ premodulations are isomorphic.

Another example of $k$-premodulation for which the isomorphism theorem holds is the classical $k$-modulation; see part (i) of the example on page 111. For $k$ modulations $\mathcal{M}=\left(F_{i},{ }_{i} M_{j}\right)$ of a valued quiver $(\mathscr{G}, \mathscr{D}, \Omega)$ and $\mathcal{M}^{\prime}=\left(F_{s}^{\prime},{ }_{s} M_{t}^{\prime}\right)$ of $\left(\mathscr{G}^{\prime}, \mathscr{D}^{\prime}, \Omega^{\prime}\right)$ with division $k$-algebras $F_{i}$ and $F_{s}^{\prime}$, denote by $T(\mathcal{M})$ and $T\left(\mathcal{M}^{\prime}\right)$ the corresponding tensor algebras as given in the proof of Proposition 3.1. Then as shown in [Liu 1991], $\mathcal{M} \cong \mathcal{M}^{\prime}$ if and only if $\mathscr{T}(M) \cong \mathscr{T}\left(M^{\prime}\right)$.

\section{Some examples from related topics}

Group species. A group species [Demonet 2010] is a triple

$$
G=\left(I,\left(\Gamma_{i}\right)_{i \in I},\left(M_{i j}\right)_{(i, j) \in I^{2}}\right)
$$

where $I$ is a finite set and $\Gamma_{i}$ is a finite group for each $i \in I$ and $M_{i j}$ is a finitedimensional $\left(k \Gamma_{i}, k \Gamma_{j}\right)$-bimodule for each $(i, j) \in I^{2}$.

A group species can be seen as a $k$-pseudomodulation of a pseudovalued quiver as follows. Consider $Q_{0}=I$ as the vertex set. For an ordered pair $(i, j) \in I \times I$, if $M_{i j} \neq 0$, set an arrow $\rho_{i j}$ from $i$ to $j$ with valuation $\left(d_{i j}, d_{j i}\right)$ for $d_{i j}=\operatorname{rank}\left({ }_{k \Gamma_{i}} M_{i j}\right)$ and $d_{j i}=\operatorname{rank}\left(M_{i j_{k \Gamma_{j}}}\right)$. Let the arrow set $Q_{1}$ consist of all such arrows. Let $\mathscr{D}=\left\{\left(d_{i j}, d_{j i}\right)\right.$ for all $\left.\rho_{i j} \in Q_{1}\right\}$. Thus, $(\mathscr{2}, \mathscr{D})$ is a pseudovalued quiver with $\mathscr{2}=$ $\left(Q_{0}, Q_{1}\right)$, and the group species $G$ can be thought as of $\left(\left(k \Gamma_{i}\right)_{i \in Q_{0}},\left(M_{i j}\right)_{(i, j) \in Q_{1}}\right)$, a pseudomodulation of $(2, \mathscr{D})$.

In [Demonet 2010], a group species $G=\left(I,\left(\Gamma_{i}\right)_{i \in I},\left(M_{i j}\right)_{(i, j) \in I^{2}}\right)$ is assumed to be over a field $k$ with char $k$ not dividing $\left|\Gamma_{i}\right|$ for $i \in I$. In this case, all $k \Gamma_{i}$ are semisimple algebras. By Theorem 4.4, we have this:

Proposition 6.1. Suppose $k$ is an algebraically closed field of characteristic 0. Then the pseudomodulation $\left(\left(k \Gamma_{i}\right)_{i \in Q_{0}},\left(M_{i j}\right)_{(i, j) \in Q_{1}}\right)$ from a group species $G=$ $\left(I,\left(\Gamma_{i}\right)_{i \in I},\left(M_{i j}\right)_{(i, j) \in I^{2}}\right)$ is a seminormal generalized modulation, where $Q_{0}=I$, $Q_{1}=\left\{(i, j) \in I^{2}: M_{i j} \neq 0\right\}$. 
As mentioned in [Demonet 2010], the category of representations of a group species is equivalent to the category of finite generated representations over its "path algebra" (that is, its tensor algebra). According to Proposition 6.1, this statement is a special case of Theorem 3.2.

The notion of group species is introduced in [Demonet 2010] with potentials and decorated representations. In some good cases, said to be nondegenerate, their mutations are defined in so that these mutations mimic the mutations of seeds defined by Fomin and Zelevinsky [2002] for a skew-symmetrizable exchange matrix defined from group species. When an exchange matrix can be associated to a nondegenerate group species with potential, an interpretation of the $F$-polynomials and the $g$-vectors in [Fomin and Zelevinsky 2007] is given in the term of the mutation of group species with potentials and their decorated representations.

Due to Proposition 6.1, we will be motivated to generalize the conclusions in [Demonet 2010] as said above to seminormal generalized modulation. In the theory of mutations, one considers a finite-dimensional basic hereditary algebra $A=k \Gamma$. Under the conditions in which the mutation can be defined, the mutation of $A$ is just isomorphic to the path algebra of the quiver that is the mutation of $\Gamma$. However, since mutations are perverse equivalent but not Morita equivalent [Ladkani 2010], it is interesting to construct the mutation theory of finite-dimensional (possibly, nonbasic) algebras via seminormal generalized modulations.

Path algebras with loops. As is well known, many lines of inquiry simplify if the underlying quivers are assumed to be without loops. See for example work on Kac's conjectures [Kac 1980; 1983; Sevenhant and Van Den Bergh 2001] and the mutation theory of basic algebras [Ladkani 2010]. One possible approach for studying quivers with loops is the viewpoint of pseudomodulations.

For a quiver $\Gamma=\left(\Gamma_{0}, \Gamma_{1}\right)$, divide the vertex set $\Gamma_{0}$ into two parts: $\Gamma_{0}=\Gamma_{0}^{0} \cup \Gamma_{0}^{1}$ where $\Gamma_{0}^{0}$ consists of all vertices without loops and $\Gamma_{0}^{1}$ consists of all vertices with loops. For a vertex $i \in \Gamma_{0}^{1}$, let $\Phi_{i}$ be the subquiver consisting of all loops at $i$. Then the whole set of loops in $\Gamma$ is just $\Phi=\bigcup_{i \in \Gamma_{0}^{1}}\left(\Phi_{i}\right)_{1}$. Define a new quiver $\breve{\Gamma}$ related to $\Gamma$ with the vertex set $\breve{\Gamma}_{0}=\Gamma_{0}$ and the arrow set $\breve{\Gamma}_{1}=\Gamma_{1} \backslash \Phi$. Clearly, this quiver $\breve{\Gamma}$ is one without loops.

The important fact is that $k \Gamma$ can be considered as a $k$-premodulation over the quiver $\breve{\Gamma}$ without loops.

In fact, define a collection $\mathscr{A}=\left\{A_{i} \mid i \in \Gamma_{0}=\breve{\Gamma}_{0}\right\}$ of $k$-algebras with $A_{i}=k$ for $i \in \Gamma_{0}^{0}$ and $A_{i}=k \Phi_{i}$ for $i \in \Gamma_{0}^{1}$; let $\breve{\Omega}(i, j)=\left\{a \in \breve{\Gamma}_{1}: t(a)=j, h(a)=i\right\}$. Then, for any $i, j \in \breve{\Gamma}_{0}$ with $i \neq j,{ }_{i} M_{j}:=A_{i} \breve{\Omega}(i, j) A_{j}$ is the free $A_{i}$ - $A_{j}$-bimodule with basis $\breve{\Omega}(i, j)$; for any $i \in \breve{\Gamma}_{0},{ }_{i} M_{i}:=0$ and $\breve{\Omega}(i, i)=\varnothing$. Thus, the path algebra $k \Gamma$ is just the generalized path algebra $k(\breve{\Gamma}, \mathscr{A})$ over the quiver $\breve{\Gamma}$ without loops.

According to Proposition 5.2, $k \Gamma=k(\breve{\Gamma}, \mathscr{A})$ is to be considered as the premod- 
ulation $\mathcal{M}=\left(A_{i},{ }_{i} M_{j}\right)$ over the valued quiver $\left(\Gamma_{0}, \mathscr{D}, \Omega\right)$ for the valuation $\mathscr{D}=$ $\left\{\left(d_{i j}, d_{j i}\right):(i, j) \in \breve{\Gamma}_{0} \times \breve{\Gamma}_{0}\right\}$, with $d_{i j}=|\breve{\Omega}(i, j)|\left|\left(\Phi_{i}\right)_{1}\right|$ and $d_{j i}=|\breve{\Omega}(i, j)|\left|\left(\Phi_{j}\right)_{1}\right|$, and the orientation $\Omega$ is given from $j$ to $i$ for any $i \neq j$ if $|\breve{\Omega}(i, j)| \neq 0$. Note that the valued quiver $\left(\Gamma_{0}, \mathscr{D}, \Omega\right)$ has no loops.

This discussion means one can transfer the study on path algebras with loops into that of generalized path algebras and premodulations of valued quivers without loops. This viewpoint gives us a new approach to those subjects whose underlying quiver has loops.

Differential tensor algebras. In [Bautista et al. 2009], the theory of differential tensor algebras is introduced as a natural generalization of the theory of algebras and their module categories. It is a useful tool in establishing some deep results in the representation theory of algebras, and has some features in common with the original theory in terms of differential graded categories as well as with formulation given in terms of bocses.

A tensor algebra $T=T(A, M)$ is given the standard grading by $T_{l}=M^{\otimes l}$ for all $l \geq 0$ with $T_{0}=A$.

For a graded $k$-algebra $T$, a linear transformation $\delta$ on $T$ is said to be a differential if it satisfies $\delta\left([T]_{i}\right) \subseteq[T]_{i+1}$ for all $i$ and the Leibniz rule $\delta(a b)=$ $\delta(a) b+(-1)^{\operatorname{deg}(a)} a \delta(b)$ for all homogeneous elements $a, b \in T$.

A differential tensor algebra or ditalgebra [Bautista et al. 2009] $\mathscr{A}$ is by definition a pair $\mathscr{A}=(T, \delta)$, where $T$ is a tensor algebra and $\delta$ is a differential on $T$ satisfying $\delta^{2}=0$.

Definition. (1) Given a $k$-pseudomodulation $\mathcal{M}=\left(A_{i},{ }_{i} M_{j}\right)_{i \in \mathscr{G}}$ of a pseudovalued quiver $(\mathscr{G}, \mathscr{D})$ and its related tensor algebra of $\mathscr{A}$-path type $T(A, M)$ as in Proposition 3.1, a linear transformation $\delta: T(A, M) \rightarrow T(A, M)$ is called a differential on $M$ if

(i) $\delta\left(A_{i}\right)$ is contained in ${ }_{i} M_{i}$;

(ii) $\delta\left({ }_{i} M_{i_{1}} \otimes_{A_{i_{1}}} \cdots \otimes_{A_{i_{s-1}} i_{s-1}} M_{j}\right)$ is contained in

$$
\begin{aligned}
& \sum_{l \in \mathscr{G}}{ }_{i} M_{l} \otimes_{A_{l} l} M_{i_{1}} \otimes_{A_{i_{1}}} \cdots \otimes_{A_{i_{s-1}} i_{s-1}} M_{j} \\
&+\sum_{l \in \mathscr{G}}{ }_{i} M_{i_{1}} \otimes_{A_{i_{1}} i_{1}} M_{l} \otimes_{A_{l} l} M_{i_{2}} \otimes_{A_{i_{2}}} \cdots \otimes_{A_{i_{s-1}} i_{s-1}} M_{j} \\
&+\cdots+\sum_{l \in \mathscr{G}}{ }_{i} M_{i_{1}} \otimes_{A_{i_{1}}} \cdots \otimes_{A_{i_{s-1}} i_{s-1}} M_{l} \otimes_{A_{l} l} M_{j} ;
\end{aligned}
$$

and the Leibniz rule $\delta(a b)=\delta(a) b+(-1)^{\operatorname{deg}(a)} a \delta(b)$ is satisfied for all $a$ in ${ }_{i} M_{i_{1}} \otimes \cdots \otimes{ }_{i_{s-1}} M_{j}$ and all $b$ in ${ }_{u} M_{u_{1}} \otimes \cdots \otimes u_{u_{t-1}} M_{v}$.

(2) A differential pseudomodulation $\mathcal{M}$ is by definition a pair $(\mathcal{M}, \delta)$ with a differential $\delta$ on $\mu$ satisfying $\delta^{2}=0$. 
It is easy to check that the Leibniz rule is satisfied by all homogeneous elements in the standard grading of $T(A, M)$. Therefore, by Proposition 3.1, the related tensor algebra $T(A, M)$ of a differential pseudomodulation $\mathcal{M}$ is a differential tensor algebra with differential $\delta$.

Differential graded category. A category $\mathcal{T}$ is called a graded category $(G C)$ [Keller 2006; Rojter 1980] if for any objects $a, b$ in $\mathscr{T}$, the set $\operatorname{Hom}_{\mathscr{T}}(a, b)$ of morphisms is a set-theoretical union of the sets $T_{i}(a, b)$ for $0 \leq i<+\infty$, and $\beta \alpha \in T_{i+j}(a, c)$ for any $\alpha \in T_{i}(a, b)$ and $\beta \in T_{j}(b, c)$, where $\alpha$ is said to be of degree $i$. If each set $T_{i}(a, b)$ is a vector space over $k$ and the multiplication by a fixed morphism is a homomorphism of these spaces, then $\mathscr{T}$ is said to be a GC over the field $k$.

For a positive integer $n$, a graded category $\mathscr{T}$ over a field $k$ is said to be a differential $n$-graded category (briefly, $n-D G C$ ) if there is a $k$-linear map $D: T \rightarrow T$ for $T=\bigoplus_{a, b \in \mathcal{T}} \operatorname{Hom}_{\mathcal{T}}(a, b)$ such that $D^{2}=0$ and $D\left(T_{i}(a, b)\right) \subseteq T_{i+n}(a, b)$ for each $a, b \in \mathscr{T}$ with $i \geq 0$, and the Leibnitz formula

$$
D(\beta \alpha)=D(\beta) \alpha+(-1)^{n \operatorname{deg} \beta} \beta D(\alpha)
$$

holds for all homogeneous elements $\alpha, \beta \in T$. This $D$ is called an $n$-differential of $\mathcal{T}$.

From [Mac Lane 1963], we know that for any bimodule $\mathcal{M}$ over a category $\mathscr{K}$, one can construct a tensor category $T(\mathcal{M})$ of $\mathcal{M}$, that is, a graded category $T(\mathcal{M})$ such that $T_{0}=\mathcal{M}, T_{1}=\mu$ and $T_{n}=M \otimes_{\mathscr{K}} \mathcal{M} \otimes_{\mathscr{K}} \cdots \otimes_{\mathscr{K}} \mathcal{M}$ with $n$ factors for $n>1$. A graded category that is a tensor algebra of a bimodule is called a semifree $G C$ in [Rojter 1980; Roiter and Kleiner 1975; Kleiner and Roiter 1977].

For an $k$-pseudomodulation $M=\left(A_{a},{ }_{a} M_{b}\right)$ of a pseudovalued quiver $(\mathscr{G}, \mathscr{D}, \Omega)$ and its related tensor algebra $T(\mathcal{M}):=T(A, M)$ of $\mathscr{A}$-path type for $A=\bigoplus_{a \in \mathscr{G}} A_{a}$ and $M=\bigoplus_{(a, b) \in \mathscr{G}_{\times} \varphi_{\mathfrak{g} a}} M_{b}$, we can define the GC $\mathcal{T}$ whose objects are the vertices in $\mathscr{G}$ and whose morphism set satisfies $\operatorname{Hom}_{\mathcal{T}}(a, b)=\bigcup_{i \geq 0} T_{i}(a, b)$ for $a, b \in \mathscr{G}$, with

$$
T_{i}(a, b)=\sum_{\left(a \alpha_{1} a_{1} \alpha_{2} a_{2} \cdots a_{i-1} \alpha_{i} b\right)}{ }_{a} M_{a_{1}} \otimes_{A_{a_{1}} a_{1}} M_{a_{2}} \otimes_{A_{a_{2}}} \cdots \otimes_{A_{a_{i-1}} a_{i-1}} M_{b}
$$

where the sum runs over all paths $\left(a \alpha_{1} a_{1} \alpha_{2} a_{2} \cdots a_{i-1} \alpha_{i} b\right)$ from $a$ to $b$ in the pseudovalued quiver $(\mathscr{G}, \mathscr{D}, \Omega)$. Trivially, $T_{i}(a, b) T_{j}(b, c) \subseteq T_{i+j}(a, c)$.

In this case, we call it a free graded category generated by the pseudovalued quiver $(\mathscr{G}, \mathscr{D}, \Omega)$ due to [Rojter 1980].

Hence, a $k$-pseudomodulation $\mathcal{M}$ and also the related tensor algebra of $\mathscr{A}$-pathtype $T(\mathcal{M})$ can equivalently be considered as this free graded category $\mathscr{T}$.

However, a differential of degree $n$ on $T(M)$ need not be a differential of some degree on its graded category $\mathcal{T}$. For example, we give in [Li and Tan 2012] for a 
path algebra $k \Gamma$ the method to construct all differentials $D$ on $k \Gamma$, not on its related graded category $\mathscr{T}_{k \Gamma}$ in general. This method requires that one finds a differential $D$ of degree $n$ on $k \Gamma$ such that the graded category $\mathscr{T}_{k \Gamma}$ of $k \Gamma$ becomes a DGC.

One is then motivated to construct differentials on a $k$-pseudomodulation $M$ and to choose them such that its corresponding graded category becomes a DGC. In general, it is interesting to characterize differentials of some degree on an arbitrary graded category and discuss the Lie algebra composed of all such differentials.

\section{Natural valued quiver and valued Ext-quiver of an algebra}

The natural quiver $\Delta_{A}$ associated to an artinian algebra $A$ is important for research in [Li 2007; $\mathrm{Li}$ and Chen 2010; Li and Lin 2012] and elsewhere.

Denote by $r$ the radical of $A$. Write $A / r=\bigoplus_{i=1}^{s} A_{i}$ where $A_{i}$ are two-sided simple ideals of $A / r$ for all $i$. Then, $r / r^{2}$ is an $A / r$-bimodule by $\bar{a} \cdot\left(x+r^{2}\right) \cdot \bar{b}=$ $a x b+r^{2}$ for any $\bar{a}=a+r, \bar{b}=b+r \in A / r$ and $x \in r$. Thus, ${ }_{i} M_{j}=A_{i} \cdot r / r^{2} \cdot A_{j}$ is a finitely generated $A_{i}-A_{j}$-bimodule for each pair $(i, j)$.

Define the vertex set $\Delta_{0}=\{1, \ldots, s\}$. For $i, j \in \Delta_{0}$, let $\operatorname{rank}\left({ }_{A_{i}}\left({ }_{i} M_{j}\right)_{A_{j}}\right)$ be the number $t_{i j}$ of arrows from $i$ to $j$ in $\Delta$. Then $\Delta_{A}=\left(\Delta_{0}, \Delta_{1}\right)$ is called the natural quiver [ $\mathrm{Li}$ and Chen 2010] of $A$. Moreover, one can construct the normal generalized path algebra $k\left(\Delta_{A}, \mathscr{A}\right)$ with $\mathscr{A}=\left\{A_{1}, \ldots, A_{s}\right\}$, which is defined as the associated normal generalized path algebra of $A$. By Proposition 5.2, we can get from $k\left(\Delta_{A}, \mathscr{A}\right)$ the corresponding normal premodulation $\mu_{A}$, which is called the corresponding normal premodulation of $A$.

For an artinian algebra $A$ and its related normal generalized algebra $k\left(\Delta_{A}, \mathscr{A}\right)$, there always exists by [Li 2007] a surjective homomorphism $\pi: k\left(\Delta_{A}, \mathscr{A}\right) \rightarrow$ $T\left(A / r, r / r^{2}\right)$ of algebras, and from the result in [Drozd and Kirichenko 1994], it follows that any such algebra $A$ with separable quotient $A / r$ is isomorphic to a quotient algebra of $k\left(\Delta_{A}, \mathscr{A}\right)$ by an admissible ideal.

An artinian algebra $A$ is said to be of Gabriel-type [Li and Lin 2012] if it is a quotient of a normal generalized path algebra. As an improvement, in [ $\mathrm{Li}$ and Lin 2012] we showed that for an artinian $k$-algebra $A$ splitting over its radical, there is a surjective algebra homomorphism $\phi: k\left(\Delta_{A}, \mathscr{A}\right) \rightarrow A$ with $J^{s} \subseteq \operatorname{ker}(\phi) \subseteq J$ for some positive integer $s$, that is, $A$ is of Gabriel type.

Moreover, we showed in [ $\mathrm{Li}$ and Lin 2012] that if an artinian algebra $A$ of Gabriel type with admissible ideal is hereditary, then $A$ is isomorphic to its related generalized path algebra $k\left(\Delta_{A}, \mathscr{A}\right)$. Hence, according to Corollary 5.4, we have:

Proposition 7.1. For a hereditary artinian algebra A of Gabriel-type with admissible ideal and its corresponding k-premodulation $\mathcal{M}=\left(A_{i},{ }_{i} M_{j}\right)$, the categories $\operatorname{Rep}(\mathcal{M})$ and $\operatorname{Mod} A$ are equivalent and so are $\operatorname{rep}(\mathcal{M})$ and $\bmod A$. 
From the discussion above, it is better if the ideal $J$ of $k\left(\Delta_{A}, \mathscr{A}\right)$ is admissible. In general, this condition is not satisfied for arbitrary nonbasic algebras. Now, we restrict to the case of basic algebras over an arbitrary field $k$, and generalize the Gabriel theorem to the case of a basic algebra that is not necessarily $k$-splitting:

Proposition 7.2. Suppose that $B$ is an artinian basic algebra with radical $r=r(B)$ over an arbitrary field $k$ and $B / r \cong F_{1} \oplus \cdots \oplus F_{s}$ for central division $k$-algebras $F_{i}$ satisfying that $\operatorname{dim}_{k} F_{i}=n_{i}^{2}$ with $\left(n_{i}, n_{j}\right)=1$ for any $i \neq j$. Then, for the associated generalized path algebra $k\left(\Delta_{B}, \mathscr{F}\right)$ of $B$ with $\mathscr{F}=\left\{F_{1}, \ldots, F_{s}\right\}$ and the natural quiver $\Delta_{B}$, there exists an admissible ideal I of $k\left(\Delta_{B}, \mathscr{F}\right)$ such that $B \cong k\left(\Delta_{B}, \mathscr{F}\right) / I$.

Proof. By the conclusion in [Pierce 1982, p. 191], $B / r$ is separable since $\operatorname{dim}_{k} F_{i}$ is finite and the center $Z\left(F_{i}\right)$ is equal to $k$ for any $i$. Due to this and [Drozd and Kirichenko 1994, Theorem 8.5.4], there is an admissible ideal $I$ of $T\left(B / r, r / r^{2}\right)$ such that $B \cong T\left(B / r, r / r^{2}\right) / I$.

Furthermore, since each $F_{i}$ is a central division algebra with $\operatorname{dim}_{k} F_{i}=n_{i}^{2}<+\infty$ and $\left(n_{i}, n_{j}\right)=1$ for any $i \neq j$, it is known from [Liu et al. 2009, p. 78] that $F_{j} \otimes F_{i}^{\mathrm{op}}$ is a central division algebra. Hence, $r / r^{2}$ is a free $F_{i}-F_{j}$-bimodule for any $i, j$. Then, according to the definition of generalized path algebra, we have $k\left(\Delta_{B}, \mathscr{F}\right) \cong T\left(B / r, r / r^{2}\right)$.

Definition. The natural valued quiver of an artinian algebra $A$ is defined to be the induced valued quiver of $k\left(\Delta_{A}, \mathscr{A}\right)$, or equivalently the valued quiver of the normal premodulation of $A$.

In the meantime, from an artinian algebra $A$, one can define another valued quiver $\left(2_{A}, \mathscr{E}, \Upsilon\right)$ [Auslander et al. 1995] as follows.

Definition. For a $k$-artinian algebra $A$, let $A / r=\bigoplus_{i=1}^{s} A_{i}$ with $A_{i} \cong M_{n_{i}}\left(D_{i}\right)$, where $D_{i}$ are division $k$-algebras for $i=1, \ldots, s$. Denote by $\left\{T_{i}\right\}_{i=1}^{s}$ the complete set of nonisomorphic simple modules of $A$. Define the valued Ext-quiver $\left(2_{A}, \mathscr{E}, \Upsilon\right)$ of $A$ as follows:

(i) $\mathscr{2}_{A}=\{1, \ldots, s\}$.

(ii) For $i, j \in 2_{A}$, draw an oriented edge from $i$ to $j$ if $\operatorname{Ext}_{A}^{1}\left(T_{j}, T_{i}\right) \neq 0$. This gives the orientation $\Upsilon$.

(iii) For $i, j \in \mathscr{Q}_{A}$, if $\operatorname{Ext}_{A}^{1}\left(T_{j}, T_{i}\right) \neq 0$, that is, there is an oriented edge from $i$ to $j$, let $e_{i j}=\operatorname{dim}_{D_{i}} \operatorname{Ext}_{A}^{1}\left(T_{j}, T_{i}\right)$ and $e_{j i}=\operatorname{dim}_{D_{j}^{\text {op }}} \operatorname{Ext}_{A}^{1}\left(T_{j}, T_{i}\right)$ and define the valuation $\mathscr{E}=\left\{\left(e_{i j}, e_{j i}\right)\right.$ for all $\left.(i, j) \in \mathscr{2}_{A} \times \mathscr{2}_{A}\right\}$.

The valued Ext-quiver is Morita invariant, but the natural valued quiver is not.

Using the notation just defined, note that $D_{i} \cong \operatorname{End}_{A}\left(T_{i}\right)$ for $i \in 2_{A}$. An artinian algebra $A$ is called $k$-splitting or splitting over the ground field $k$ if $D_{i} \cong k$ for each $i$. 
For example, $A$ is always $k$-splitting if the ground field $k$ is algebraically closed.

When $A$ is $k$-splitting, the valued Ext-quiver of $A$ degenerates to a nonvalued quiver, which is just the Ext-quiver of $A$. In this case, we have the following results from [Li and Lin 2012]:

First, the vertex set of the Ext-quiver of $A$ equals that of the natural quiver of $A$.

Second, $t_{i j}=\left\lceil m_{i j} /\left(n_{i} n_{j}\right)\right\rceil$, where $t_{i j}$ and $m_{i j}$ are respectively the arrow numbers of the natural quiver and the Ext-quiver of $A$ from $i$ to $j$, and $n_{i}=\operatorname{dim}_{k} T_{i}$ for the irreducible module $T_{i}$ of $A$ at the vertex $i$.

Third, if $A$ is a basic algebra, then the Ext-quiver is just the natural quiver.

Now, their analogues will be given in the case that $A$ is non- $k$-splitting in general.

Lemma 7.3. Let $A$ be an artinian algebra with radical $r$ such that $A / r=\bigoplus_{i=1}^{s} A_{i}$, where $A_{i} \cong M_{n_{i}}\left(F_{i}\right)$ for division k-algebras $F_{i}$ for $i=1, \ldots, s$. Let $\left\{u_{i}\right\}_{i=1}^{s}$ be the complete set of primitive orthogonal idempotents of $A$ and $\left\{T_{i}\right\}_{i=1}^{s}$ be the corresponding complete set of nonisomorphic A-simple modules. Then

$$
\operatorname{dim}_{k}\left(u_{i} r / r^{2} u_{j}\right)=\operatorname{dim}_{k} \operatorname{Ext}_{A}^{1}\left(T_{j}, T_{i}\right) \quad \text { for } i, j \in\{1, \ldots, s\} .
$$

Proof. For $i, j=1, \ldots, s$, let $P_{j} \rightarrow T_{j}$ be a projective cover. Then there is the exact sequence $0 \rightarrow r P_{j} \rightarrow P_{j} \rightarrow T_{j} \rightarrow 0$. Applying the functor $\operatorname{Hom}_{A}\left(-, T_{i}\right)$, we obtain this exact sequence of $k$-linear spaces:

$$
0 \rightarrow \operatorname{Hom}_{A}\left(T_{j}, T_{i}\right) \rightarrow \operatorname{Hom}_{A}\left(P_{j}, T_{i}\right) \stackrel{h}{\rightarrow} \operatorname{Hom}_{A}\left(r P_{j}, T_{i}\right) \rightarrow \operatorname{Ext}_{A}^{1}\left(T_{j}, T_{i}\right) \rightarrow 0 .
$$

By Schur's lemma, $\operatorname{Hom}_{A}\left(T_{j}, T_{i}\right)=F_{j}$ if $i=j$ and vanishes otherwise. Since $T_{j} \cong P_{j} / r P_{j}$ and $r T_{i}=0$ for any $i, j$, it follows that $h$ must be the zero map for $i \neq j$. Hence we have

$$
\operatorname{Hom}_{A}\left(r P_{j}, T_{i}\right) \cong \operatorname{Ext}_{A}^{1}\left(T_{j}, T_{i}\right) .
$$

On the other hand,

$$
\operatorname{Hom}_{A}\left(r P_{j}, T_{i}\right)=\operatorname{Hom}_{A}\left(r P_{j} / r^{2} P_{j}, T_{i}\right) \cong \operatorname{Hom}_{A} / r\left(r P_{j} / r^{2} P_{j}, T_{i}\right) .
$$

Since $A / r$ is semisimple, $r P_{j} / r^{2} P_{j}$ is a direct sum of some $T_{p}$ as $A / r$-modules. Thus,

(6) $\operatorname{Hom}_{A / r}\left(r P_{j} / r^{2} P_{j}, T_{i}\right) \cong \operatorname{Hom}_{A / r}\left(T_{i}, r P_{j} / r^{2} P_{j}\right) \cong \operatorname{Hom}_{A}\left(P_{i}, r P_{j} / r^{2} P_{j}\right)$.

Using $P_{j}=A u_{j}$ for any $j$ and [Auslander et al. 1995, Proposition I.4.9], we have $\operatorname{Hom}_{A}\left(P_{i}, r^{m} P_{j}\right) \cong u_{i} r^{m} u_{j}$ for any positive integer $m$. Via these isomorphisms for $m=1,2$, we can get $\operatorname{Hom}_{A}\left(P_{i}, r P_{j} / r^{2} P_{j}\right) \cong u_{i} r / r^{2} u_{j}$. Using this, (4), (5) and (6), we get $u_{i} r / r^{2} u_{j} \cong \operatorname{Ext}_{A}^{1}\left(T_{j}, T_{i}\right)$ as $k$-linear spaces. 
This lemma's assumptions differ from those in [Auslander et al. 1995, Proposition III.1.14] in that the ground field $k$ is arbitrary and $A$ is not assumed to be basic.

Now, we give the relationship between the natural valued quiver and the Extvalued quiver for a basic algebra $B$.

Two valued quivers $(\mathscr{G}, \mathscr{D}, \Omega)$ and $(2, \mathscr{E}, \Upsilon)$ are called pair-opposite equal if $\mathscr{G}=2$ and $\Omega=\Upsilon$ and if $d_{i j}=e_{j i}$ and $d_{j i}=e_{i j}$ for any $\left(d_{i j}, d_{j i}\right) \in \mathscr{D},\left(e_{i j}, e_{j i}\right) \in \mathscr{E}$.

From this definition, we think that two pair-opposite equal valued quivers are indeed equal under rewriting the order of pairs of valuation.

For the radical $r$ of $B$, we have $B / r \cong F_{1} \oplus \cdots \oplus F_{s}$ for division $k$-algebras $F_{i}$.

The normal regular modulation $\mathcal{M}=\left(F_{i},{ }_{i} M_{j}\right)$ is constructed from $k\left(\Delta_{B}, \mathscr{F}\right)$ with ${ }_{i} M_{j}=F_{i}\left(r / r^{2}\right) F_{j}$ as $F_{i}-F_{j}$-bimodules for any $i, j \in \Delta_{0}$.

The natural valued quiver of $B$, that is, the induced valued quiver from $k\left(\Delta_{B}, \mathscr{F}\right)$, is $\left(\Delta_{0}, \mathscr{D}, \Omega\right)$ with a unique oriented edge from $i$ to $j$ when ${ }_{i} M_{j} \neq 0$ and $\mathscr{D}=$ $\left\{\left(d_{i j}, d_{j i}\right):(i, j) \in \Delta_{0} \times \Delta_{0}\right\}$ for

$$
d_{i j}=\operatorname{dim}\left({ }_{i} M_{j}\right)_{F_{j}}=t_{i j} \varepsilon_{i} \quad \text { and } \quad d_{j i}=\operatorname{dim}_{F_{i}}\left({ }_{i} M_{j}\right)=t_{i j} \varepsilon_{j},
$$

with $t_{i j}=\operatorname{dim}_{\left(F_{j}^{\mathrm{op}} \otimes F_{i}\right)} M_{j}$ the arrow number from $i$ to $j$ in $\Delta_{B}$, and $\varepsilon_{i}=\operatorname{dim}_{k} F_{i}$, $\varepsilon_{j}=\operatorname{dim}_{k} F_{j}$ satisfying $d_{i j} \varepsilon_{j}=d_{j i} \varepsilon_{i}$.

Theorem 7.4. The natural valued quiver $\left(\Delta_{0}, \mathscr{D}, \Omega\right)$ and the valued Ext-quiver $(2, \mathscr{E}, \Upsilon)$ of an artinian basic $k$-algebra $B$ are pair-opposite equal.

Proof. First, $\Delta_{0}=2=\{1, \ldots, s\}$.

By Lemma 7.3, there is an oriented edge from $i$ to $j$ in $\left(\Delta_{0}, \mathscr{D}, \Omega\right)$ if and only if there is an oriented edge from $i$ to $j$ in $(2, \mathscr{E}, \Upsilon)$, that is, $\Omega=\Upsilon$.

In the natural quiver $\Delta_{B}$ of $B$ the arrow number $t_{i j}$ is equal to $\operatorname{dim}_{F_{j}^{\mathrm{op}} \otimes F_{i}} M_{j}$ for any $i, j \in \Delta_{0}$.

Let $m_{i j}=\operatorname{dim}_{k}\left(u_{i} r / r^{2} u_{j}\right)$. We have $u_{i} r / r^{2} u_{j}=F_{i}\left(r / r^{2}\right) F_{j}={ }_{i} M_{j}$. Then

$$
m_{i j}=\operatorname{dim}_{F_{j}^{\mathrm{op}} \otimes F_{i}}\left({ }_{i} M_{j}\right) \operatorname{dim}_{k}\left(F_{j}^{\mathrm{op}} \otimes F_{i}\right)=t_{i j} \varepsilon_{i} \varepsilon_{j}
$$

Thus

$$
\begin{aligned}
\operatorname{dim}_{k} \operatorname{Ext}_{B}^{1}\left(T_{j}, T_{i}\right) & =\operatorname{dim}_{\operatorname{End}_{B}\left(T_{i}\right)} \operatorname{Ext}_{B}^{1}\left(T_{j}, T_{i}\right) \operatorname{dim}_{k} \operatorname{End}_{B}\left(T_{i}\right) \\
& =e_{i j} \operatorname{dim}_{k} F_{i}=e_{i j} \varepsilon_{i} .
\end{aligned}
$$

By Lemma 7.3 and (8), $m_{i j}=e_{i j} \varepsilon_{i}$. Similarly, it is given that $m_{i j}=e_{j i} \varepsilon_{j}$.

By (7) and $d_{i j}=t_{i j} \varepsilon_{i}$ and $d_{j i}=t_{i j} \varepsilon_{j}$, we get that $d_{j i} \varepsilon_{i}=t_{i j} \varepsilon_{j} \varepsilon_{i}=m_{i j}=e_{i j} \varepsilon_{i}$. Thus, $d_{j i}=e_{i j}$. Similarly, $d_{i j}=e_{j i}$.

In summary, $\left(\Delta_{0}, \mathscr{D}, \Omega\right)$ and $(2, \mathscr{E}, \Upsilon)$ are pair-opposite equal.

We think this consequence is evidence for the notion of natural valued quiver of an artinian algebra $A$ as given above. 
By definition, a natural quiver and a natural valued quiver can be constructed from each other. In general, natural valued quivers, as well as natural quivers, would change under Morita equivalence between algebras.

Now, we discuss the relation between the natural valued quiver $\left(\left(\Delta_{0}\right)_{A}, \mathscr{D}, \Omega\right)$ and the valued Ext-quiver $\left(2_{A}, \mathscr{E}, \Upsilon\right)$ for an arbitrary artinian algebra $A$.

Theorem 7.5. For an artinian algebra $A$, the natural valued quiver $\left(\left(\Delta_{0}\right)_{A}, \mathscr{D}, \Omega\right)$ and the valued Ext-quiver $\left(\mathscr{Q}_{A}, \mathscr{E}, \Upsilon\right)$ satisfy the following relations.

(i) The vertex sets are equal, that is, $\left(\Delta_{0}\right)_{A}=2_{A}$.

(ii) The orientations are the same, that is, $\Omega=\Upsilon$.

(iii) The valuations $\mathscr{D}=\left\{\left(d_{i j}, d_{j i}\right):(i, j) \in\left(\Delta_{0}\right)_{A} \times\left(\Delta_{0}\right)_{A}\right\}$ and $\mathscr{E}=\left\{\left(e_{i j}, e_{j i}\right)\right.$ : $\left.(i, j) \in 2_{A} \times \mathscr{2}_{A}\right\}$ satisfy the formulas

$$
d_{j i}=e_{i j} n_{j}^{2} \frac{t_{i j}}{m_{i j}} \quad \text { and } \quad d_{i j}=e_{j i} n_{i}^{2} \frac{t_{i j}}{m_{i j}}
$$

for any vertices $i, j$. Here, $t_{i j}$ is the arrow number in the natural quiver $\Delta_{A}$ of $A$ from $i$ to $j, m_{i j}$ is the arrow number in the natural quiver $\Delta_{B}$ of the associated basic algebra $B$ of A from $i$ to $j$ and $n_{i}=\operatorname{dim}_{k} S_{i} /\left(\operatorname{dim}_{k}\right.$ End $\left.S_{i}\right)$ for the simple module $S_{i}$ of $A$ at the vertex $i$.

Proof. (i) This is easy due to their definitions.

(ii) By Lemma 7.3, ${ }_{i} M_{j}=A_{i}\left(r / r^{2}\right) A_{j} \neq 0$ if and only if $u_{i} r / r^{2} u_{j} \neq 0$ if and only if $\operatorname{Ext}_{A}^{1}\left(T_{j}, T_{i}\right) \neq 0$. Then, the claim follows from the definitions of the orientations $\Omega$ and $\Upsilon$.

(iii) By the proof of Lemma 5.1, $d_{i j}=t_{i j} \varepsilon_{i}$ and $d_{j i}=t_{i j} \varepsilon_{j}$, where $t_{i j}$ is the arrow number in $\Delta_{A}$ from $i$ to $j$, and $\varepsilon_{i}=\operatorname{dim}_{k} A_{i}$ for $A / r_{A}=A_{1} \oplus \cdots \oplus A_{s}$.

First, the valued Ext-quiver $\left(2_{A}, \mathscr{E}, \Upsilon\right)$ of $A$ is equal to that of its associated basic algebra $B$. And, by Theorem 7.4, the latter is pair-opposite equal to the natural valued quiver $\left(\left(\Delta_{0}\right)_{B}, \mathscr{D}_{B}, \Omega_{B}\right)$ of $B$. Hence, $\left(\mathscr{2}_{A}, \mathscr{E}, \Upsilon\right)$ is pair-opposite equal to $\left(\left(\Delta_{0}\right)_{B}, \mathscr{D}_{B}, \Omega_{B}\right)$. Therefore, for $\mathscr{E}=\left\{\left(e_{i j}, e_{j i}\right):(i, j) \in \mathscr{2}_{A} \times \mathscr{Q}_{A}\right\}$ and $\mathscr{D}_{B}=\left\{\left(d_{i j}^{B}, d_{j i}^{B}\right):(i, j) \in\left(\Delta_{0}\right)_{A} \times\left(\Delta_{0}\right)_{A}\right\}$, it follows that

$$
e_{i j}=d_{j i}^{B}=m_{i j} \varepsilon_{j}^{B} \quad \text { and } \quad e_{j i}=d_{i j}^{B}=m_{i j} \varepsilon_{i}^{B},
$$

where $m_{i j}$ is the arrow number in the natural quiver $\Delta_{B}$ of $B$ from $i$ to $j$ and $\varepsilon_{i}^{B}=\operatorname{dim}_{k} F_{i}$ for $B / r_{B}=F_{1} \oplus \cdots \oplus F_{s}$ with division $k$-algebras $F_{i} \cong$ End $S_{i}$ for the simple module $S_{i}$ of $A$ at the vertex $i$ for $i=1, \ldots, s$.

Due to the Wedderburn-Artin theorem, $A_{i} \cong M_{n_{i}}(k) \otimes F_{i}$ for any $i$, where $n_{i}=\operatorname{dim}_{k} S_{i} /\left(\operatorname{dim}_{k}\right.$ End $\left.S_{i}\right)$ is a positive integer. Then we get

$$
d_{j i}=t_{i j} n_{j}^{2} \varepsilon_{j}^{B} \quad \text { and } \quad d_{i j}=t_{i j} n_{i}^{2} \varepsilon_{i}^{B}
$$


By (10) and (11), it follows that $d_{j i}=e_{i j} n_{j}^{2} t_{i j} / m_{i j}$ and $d_{i j}=e_{j i} n_{i}^{2} t_{i j} / m_{i j}$.

Obviously, Theorem 7.4 is just the special case of Theorem 7.5 when $A$ is basic. By the formula given in [Li and Lin 2012], when $A$ is $k$-splitting, we have $t_{i j}=\left\lceil m_{i j} /\left(n_{i} n_{j}\right)\right\rceil$. Then, in this case, formula (9) gives us this corollary:

Corollary 7.6. Let A be a $k$-splitting artinian algebra, and adopt the notation of Theorem 7.5. Then for any vertices $i, j$,

$$
d_{j i}=e_{i j} n_{j}^{2} \frac{1}{m_{i j}}\left\lceil\frac{m_{i j}}{n_{i} n_{j}}\right\rceil \text { and } d_{i j}=e_{j i} n_{i}^{2} \frac{1}{m_{i j}}\left\lceil\frac{m_{i j}}{n_{i} n_{j}}\right\rceil .
$$

\section{References}

[Auslander et al. 1995] M. Auslander, I. Reiten, and S. O. Smalø, Representation theory of Artin algebras, Cambridge Studies in Adv. Math. 36, Cambridge Univ. Press, 1995. MR 96c:16015

[Bautista et al. 2009] R. Bautista, L. Salmerón, and R. Zuazua, Differential tensor algebras and their module categories, London Mathematical Society Lecture Note Series 362, Cambridge Univ. Press, 2009. MR 2011m:16016 Zbl 05610895

[Chen 2008] L. L. Chen, Quivers and Hopfalgebras, Ph.D. thesis, Zhejiang University, China, 2008.

[Coelho and Liu 2000] F. U. Coelho and S.-X. Liu, "Generalized path algebras", pp. 53-66 in Interactions between ring theory and representations of algebras, edited by F. Van Oystaeyen and M. Saorin, Lecture Notes in Pure and Appl. Math. 210, Dekker, New York, 2000. MR 2001c:16027 Zbl 0987.16007

[Demonet 2010] L. Demonet, "Mutations of group species with potentials and their representations: Applications to cluster algebras", preprint, 2010. arXiv 1003.5078v2

[Dlab 1980] V. Dlab, Representations of valued graphs, Séminaire de Mathématiques Supérieures 73, Presses de l'Université de Montréal, Montreal, 1980. MR 82k:16037 Zbl 0478.16026

[Dlab and Ringel 1976] V. Dlab and C. M. Ringel, Indecomposable representations of graphs and algebras, Mem. Amer. Math. Soc. 173, Amer. Math. Soc., Providence, RI, 1976. MR 56 \#5657 Zbl 0332.16015

[Drozd and Kirichenko 1994] Y. A. Drozd and V. V. Kirichenko, Finite-dimensional algebras, Springer, Berlin, 1994. MR 95i:16001 Zbl 0816.16001

[Fomin and Zelevinsky 2002] S. Fomin and A. Zelevinsky, "Cluster algebras, I: Foundations", $J$. Amer. Math. Soc. 15:2 (2002), 497-529. MR 2003f:16050 Zbl 1021.16017

[Fomin and Zelevinsky 2007] S. Fomin and A. Zelevinsky, "Cluster algebras, IV: Coefficients", Compos. Math. 143:1 (2007), 112-164. MR 2008d:16049 Zbl 1127.16023

[Kac 1980] V. G. Kac, "Infinite root systems, representations of graphs and invariant theory", Invent. Math. 56:1 (1980), 57-92. MR 82j:16050 Zbl 0427.17001

[Kac 1983] V. G. Kac, "Root systems, representations of quivers and invariant theory", pp. 74108 in Invariant theory (Montecatini, 1982), edited by F. Gherardelli, Lecture Notes in Math. 996, Springer, Berlin, 1983. MR 85j:14088 Zbl 0534.14004

[Keller 2006] B. Keller, "On differential graded categories", pp. 151-190 in International Congress of Mathematicians, vol. 2, edited by M. Sanz-Solé et al., Eur. Math. Soc., Zürich, 2006. MR 2008g:18015 Zbl 1140.18008

[Kleiner and Roiter 1977] M. M. Kleĭner and A. V. Roĭter, "Representations of differential graded categories", pp. 5-70 in Matrix problems, edited by L. A. Nazarova, Akad. Nauk Ukrain. SSR Inst. Mat., Kiev, 1977. In Russian. MR 58 \#28129 Zbl 0496.16038 
[Ladkani 2010] S. Ladkani, "Perverse equivalences, BB-tilting, mutations and applications", preprint, 2010. arXiv 1001.4765

[Li 2007] F. Li, "Characterization of left Artinian algebras through pseudo path algebras", J. Aust. Math. Soc. 83:3 (2007), 385-416. MR 2009f:16022 Zbl 1156.16015

[Li and Chen 2010] F. Li and L. Chen, "The natural quiver of an Artinian algebra", Algebr. Represent. Theory 13:5 (2010), 623-636. MR 2011f:16033 Zbl 05818582

[Li and Lin 2012] F. Li and Z. Lin, "Approach to Artinian algebras via natural quivers", Trans. Amer. Math. Soc. 364:3 (2012), 1395-1411. MR 2869180 Zbl 06024131

[Li and Liu 2008] F. Li and G. X. Liu, "Generalized path coalgebras and a generalized dual Gabriel theorem”, Acta Math. Sinica (Chin. Ser.) 51:5 (2008), 853-862. MR 2009k:16074 Zbl 1174.16007

[Li and Tan 2012] F. Li and D. Tan, "The graded Lie algebra of grading differential operators on a path algebra", 2012. Submitted to Homology Homotopy Appl.

[Li and Wen 2008] F. Li and D. Wen, "Ordinary quiver, AR-quiver and natural quiver of an algebra", pp. 271-304 in Geometry, analysis and topology of discrete groups (Beijing, 2006), edited by L. Ji et al., Adv. Lect. Math. (ALM) 6, Int. Press, Somerville, MA, 2008. MR 2009k:16024 Zbl 1172.16009

[Liu 1991] S. X. Liu, "Isomorphism problem for tensor algebras over valued graphs", Sci. China Ser. A 34:3 (1991), 267-272. MR 92b:16054 Zbl 0735.16007

[Liu et al. 1986] S. X. Liu, Y. L. Luo, and J. Xiao, "Isomorphisms of path algebras", Beijing Shifan Daxue Xuebao 31:3 (1986), 13-20. MR 869867 Zbl 0643.16016

[Liu et al. 2009] S. X. Liu, J. Y. Guo, B. Zhu, and Y. Han, Rings and Algebras, Science Press, Beijing, 2009.

[Mac Lane 1963] S. Mac Lane, Homology, Die Grundlehren der mathematischen Wissenschaften 114, Academic Press, Publishers, New York, 1963. MR 28 \#122 Zbl 0328.18009

[Pierce 1982] R. S. Pierce, Associative algebras, Graduate Texts in Mathematics 88, Springer, New York, 1982. MR 84c:16001 Zbl 0497.16001

[Roiter and Kleiner 1975] A. V. Roŭter and M. M. Kleiner, "Representations of differential graded categories", pp. 316-339 in Representations of algebras (Ottawa, 1974), edited by V. Dlab, Lecture Notes in Math. 488, Springer, Berlin, 1975. MR 55 \#8106 Zbl 0356.16011

[Rojter 1980] A. V. Rojter, "Matrix problems and representations of BOCSs", pp. 288-324 in Representation theory, I (Ottawa, 1979), edited by V. Dlab and P. Gabriel, Lecture Notes in Math. 831, Springer, Berlin, 1980. MR 83e:16034 Zbl 0473.18006

[Sevenhant and Van Den Bergh 2001] B. Sevenhant and M. Van Den Bergh, "A relation between a conjecture of Kac and the structure of the Hall algebra”, J. Pure Appl. Algebra 160:2-3 (2001), 319-332. MR 2002f:17031 Zbl 1025.17006

Received March 30, 2011. Revised November 7, 2011.

FANG LI

DEPARTMENT OF MATHEMATICS

ZHEJIANG UNIVERSITY

YUQUAN CAMPUS

HANGZHOU, 310027

CHINA

fangli@zju.edu.cn 


\title{
PACIFIC JOURNAL OF MATHEMATICS
}

\author{
http://pacificmath.org \\ Founded in 1951 by \\ E. F. Beckenbach (1906-1982) and F. Wolf (1904-1989)
}

\section{EDITORS}

V. S. Varadarajan (Managing Editor)

Department of Mathematics

University of California

Los Angeles, CA 90095-1555

pacific@math.ucla.edu

Vyjayanthi Chari

Department of Mathematics

University of California

Riverside, CA 92521-0135

chari@math.ucr.edu

\section{Robert Finn}

Department of Mathematics Stanford University

Stanford, CA 94305-2125

finn@math.stanford.edu

Kefeng Liu

Department of Mathematics

University of California

Los Angeles, CA 90095-1555

liu@math.ucla.edu
Darren Long

Department of Mathematics

University of California

Santa Barbara, CA 93106-3080

long@math.ucsb.edu

Jiang-Hua Lu

Department of Mathematics

The University of Hong Kong

Pokfulam Rd., Hong Kong jhlu@maths.hku.hk

Alexander Merkurjev

Department of Mathematics

University of California

Los Angeles, CA 90095-1555

merkurev@math.ucla.edu
Sorin Popa

Department of Mathematics University of California

Los Angeles, CA 90095-1555 popa@math.ucla.edu

Jie Qing

Department of Mathematics

University of California

Santa Cruz, CA 95064

qing@cats.ucsc.edu

Jonathan Rogawski

Department of Mathematics

University of California

Los Angeles, CA 90095-1555

jonr@math.ucla.edu

\section{PRODUCTION}

pacific@math.berkeley.edu

\section{SUPPORTING INSTITUTIONS}

ACADEMIA SINICA, TAIPEI

CALIFORNIA INST. OF TECHNOLOGY INST. DE MATEMÁTICA PURA E APLICADA KEIO UNIVERSITY

MATH. SCIENCES RESEARCH INSTITUTE NEW MEXICO STATE UNIV.

OREGON STATE UNIV.

\author{
STANFORD UNIVERSITY \\ UNIV. OF BRITISH COLUMBIA \\ UNIV. OF CALIFORNIA, BERKELEY \\ UNIV. OF CALIFORNIA, DAVIS \\ UNIV. OF CALIFORNIA, LOS ANGELES \\ UNIV. OF CALIFORNIA, RIVERSIDE \\ UNIV. OF CALIFORNIA, SAN DIEGO \\ UNIV. OF CALIF., SANTA BARBARA
}

\author{
UNIV. OF CALIF., SANTA CRUZ \\ UNIV. OF MONTANA \\ UNIV. OF OREGON \\ UNIV. OF SOUTHERN CALIFORNIA \\ UNIV. OF UTAH \\ UNIV. OF WASHINGTON \\ WASHINGTON STATE UNIVERSITY
}

These supporting institutions contribute to the cost of publication of this Journal, but they are not owners or publishers and have no responsibility for its contents or policies.

See inside back cover or pacificmath.org for submission instructions.

The subscription price for 2012 is US \$420/year for the electronic version, and \$485/year for print and electronic.

Subscriptions, requests for back issues from the last three years and changes of subscribers address should be sent to Pacific Journal of Mathematics, P.O. Box 4163, Berkeley, CA 94704-0163, U.S.A. Prior back issues are obtainable from Periodicals Service Company, 11 Main Street, Germantown, NY 12526-5635. The Pacific Journal of Mathematics is indexed by Mathematical Reviews, Zentralblatt MATH, PASCAL CNRS Index, Referativnyi Zhurnal, Current Mathematical Publications and the Science Citation Index.

The Pacific Journal of Mathematics (ISSN 0030-8730) at the University of California, c/o Department of Mathematics, 969 Evans Hall, Berkeley, CA 94720-3840, is published monthly except July and August. Periodical rate postage paid at Berkeley, CA 94704, and additional mailing offices. POSTMASTER: send address changes to Pacific Journal of Mathematics, P.O. Box 4163, Berkeley, CA 94704-0163.

PJM peer review and production are managed by EditFLOW ${ }^{\mathrm{TM}}$ from Mathematical Sciences Publishers.

PUBLISHED BY PACIFIC JOURNAL OF MATHEMATICS

at the University of California, Berkeley 94720-3840

A NON-PROFIT CORPORATION

Typeset in LATEX

Copyright $(02012$ by Pacific Journal of Mathematics 


\section{PACIFIC JOURNAL OF MATHEMATICS}

Volume $256 \quad$ No. $1 \quad$ March 2012

On slim double Lie groupoids

NiCOlas ANDruskiewitsch, JEsus OCHOA ARANGO and AlEJANDRO

TIRABOSCHI

Topological classification of quasitoric manifolds with second Betti number 2

19

Suyoung Choi, SeOnjeOng PARK and Dong Youp SuH

Refined Kato inequalities for harmonic fields on Kähler manifolds

DANiEL Cibotaru and PENG ZHU

Deformation retracts to the fat diagonal and applications to the existence of peak solutions of nonlinear elliptic equations

E. Norman Dancer, Jonathan Hillman and Angela Pistoia

Descent for differential Galois theory of difference equations: confluence and $q$-dependence

LuCia Di Vizio and Charlotte HaRdouin

Modulation and natural valued quiver of an algebra

FANG LI

Willmore hypersurfaces with two distinct principal curvatures in $\mathbb{R}^{n+1}$

TONGZHU LI

Variational inequality for conditional pressure on a Borel subset

YuAN Li, ERCAI CHEN and WEN-ChIAO CHENG

New homotopy 4-spheres

DANIEL NASH

Combinatorial constructions of three-dimensional small covers

YASUZO NISHIMURA

On a theorem of Paul Yang on negatively pinched bisectional curvature

AERYEONG SEO

Orders of elements in finite quotients of Kleinian groups

PETER B. Shalen

A new algorithm for finding an 1.c.r. set in certain two-sided cells

JIAN-YI SHI

Addendum to the article Superconnections and parallel transport

FLORIN DUMITRESCU 